\title{
Homogeneous analysis of globular clusters from the APOGEE survey with the BACCHUS code
}

\section{The northern clusters ${ }^{\star}$}

T. Masseron ${ }^{1,2}$, D. A. García-Hernández ${ }^{1,2}$, Sz. Mészáros ${ }^{3,4}$, O. Zamora ${ }^{1,2}$, F. Dell'Agli $^{1,2}$, C. Allende Prieto ${ }^{1,2}$, B. Edvardsson ${ }^{5}$, M. Shetrone ${ }^{6}$, B. Plez ${ }^{7}$, J. G. Fernández-Trincado ${ }^{8,9,10}$, K. Cunha ${ }^{11,12}$, H. Jönsson ${ }^{13}$, D. Geisler ${ }^{9,14,15}$, T. C. Beers ${ }^{16}$, and R. E. Cohen ${ }^{17}$

${ }^{1}$ Instituto de Astrofísica de Canarias, 38205 La Laguna, Tenerife, Spain e-mail: tmasseron@iac.es

2 Departamento de Astrofísica, Universidad de La Laguna, 38206 La Laguna, Tenerife, Spain

3 ELTE Eötvös Loránd University, Gothard Astrophysical Observatory, Szombathely, Hungary

${ }^{4}$ Premium Postdoctoral Fellow of the Hungarian Academy of Sciences, Hungary

5 Theoretical Astrophysics, Department of Physics and Astronomy, Uppsala University, Box 516, 75120 Uppsala, Sweden

${ }^{6}$ McDonald Observatory, University of Texas at Austin, Fort Davis, TX 79734, USA

7 Laboratoire Univers et Particules de Montpellier, Université de Montpellier, CNRS, 34095 Montpellier Cedex 05, France

8 Instituto de Astronomía y Ciencias Planetarias, Universidad de Atacama, Copayapu 485 Copiapó, Chile

9 Departamento de Astronomía, Casilla 160-C, Universidad de Concepción, Concepción, Chile

10 Institut Utinam, CNRS UMR 6213, Université Bourgogne-Franche-Comté, OSU THETA Franche-Comté, Observatoire de Besançon, BP 1615, 25010 Besançon Cedex, France

11 Observatório Nacional/MCTI, Rua Gen. José Cristino 77, 20921-400 Rio de Janeiro, Brazil

12 Steward Observatory, University of Arizona Tucson, 85719, USA

13 Lund Observatory, Department of Astronomy and Theoretical Physics, Lund University, Box 43, 22100 Lund, Sweden

14 Instituto de Investigación Multidisciplinario en Ciencia y Tecnología, Universidad de La Serena, Avenida Raúl Bitrán s/n, La Serena, Chile

15 Departamento de Física y Astronomía, Facultad de Ciencias, Universidad de La Serena, Av. Juan Cisternas, 1200 La Serena, Chile

16 Department of Physics and JINA Center for the Evolution of the Elements, University of Notre Dame, Notre Dame, IN 46556, USA

17 Space Telescope Science Institute, 3700 San Martin Drive, Baltimore, MD 21210, USA

Received 31 October 2018 / Accepted 20 December 2018

\section{ABSTRACT}

\begin{abstract}
Aims. We seek to provide abundances of a large set of light and neutron-capture elements homogeneously analyzed that cover a wide range of metallicity to constrain globular cluster (GC) formation and evolution models.

Methods. We analyzed a large sample of 885 GCs giants from the SDSS IV-Apache Point Observatory Galactic Evolution Experiment (APOGEE) survey. We used the Cannon results to separate the red giant branch and asymptotic giant branch stars, not only allowing for a refinement of surface gravity from isochrones, but also providing an independent $H$-band spectroscopic method to distinguish stellar evolutionary status in clusters. We then used the Brussels Automatic Code for Characterizing High accUracy Spectra (BACCHUS) to derive metallicity, microturbulence, macroturbulence, many light-element abundances, and the neutron-capture elements $\mathrm{Nd}$ and $\mathrm{Ce}$ for the first time from the APOGEE GCs data.

Results. Our independent analysis helped us to diagnose issues regarding the standard analysis of the APOGEE DR14 for lowmetallicity GC stars. Furthermore, while we confirm most of the known correlations and anticorrelation trends ( $\mathrm{Na}-\mathrm{O}, \mathrm{Mg}-\mathrm{Al}, \mathrm{C}-\mathrm{N})$, we discover that some stars within our most metal-poor clusters show an extreme Mg depletion and some Si enhancement. At the same time, these stars show some relative Al depletion, displaying a turnover in the Mg-Al diagram. These stars suggest that $\mathrm{Al}$ has been partially depleted in their progenitors by very hot proton-capture nucleosynthetic processes. Furthermore, we attempted to quantitatively correlate the spread of Al abundances with the global properties of GCs. We find an anticorrelation of the Al spread against clusters metallicity and luminosity, but the data do not allow us to find clear evidence of a dependence of $\mathrm{N}$ against metallicity in the more metal-poor clusters.

Conclusions. Large and homogeneously analyzed samples from ongoing spectroscopic surveys unveil unseen chemical details for many clusters, including a turnover in the $\mathrm{Mg}$ - $\mathrm{Al}$ anticorrelation, thus yielding new constrains for GCs formation/evolution models.
\end{abstract}

Key words. stars: abundances - globular clusters: general

\footnotetext{
^ Full Table 2 is only available at the CDS via anonymous ftp to cdsarc.u-strasbg. fr $(130.79 .128 .5)$ or via http://cdsarc.u-strasbg.fr/viz-bin/qcat?J/A+A/622/A191
} 


\section{Introduction}

The existence of multiple populations in globular clusters (GC) can be unambiguously observed in appropriate color-magnitude diagrams (e.g., Milone et al. 2017, and reference therein) and the variations in colors are associated with abundances variations (see, e.g., Monelli et al. 2013; Mészáros et al. 2018). The color indices sensitive to multiple populations have such a sensitivity because their band-pass includes the spectral features that are changing, for example $\mathrm{C}_{\mathrm{UBI}}$ or the Milone et al. "magic trio" with WFC3 UVIS. Some broadband colors (V-I in most cases) are mostly insensitive to such $(\mathrm{C}, \mathrm{N}, \mathrm{O}$, etc.) variations, but most color indices are sensitive to metallicity in various ways. Indeed, it has been known for a long time that some elemental abundances vary from star to star within the clusters. The most observed elements showing abundance variations are $\mathrm{C}, \mathrm{N}, \mathrm{O}$, Mg, Na, and Al (Smith 1987; Kraft 1994; Gratton et al. 2004, 2012, see past reviews and references therein). Si has been more recently revealed to vary in some clusters (e.g., Yong et al. 2005; Carretta et al. 2009a). Ca is another element that has been revealed to show a spread in some clusters (Marino et al. 2009). Finally, $\mathrm{K}$ has been observed to vary in only two clusters: NGC 2808 and NGC 2419 (Cohen \& Kirby 2012; Mucciarelli et al. 2015a). Regarding neutron capture elements, a few clusters show significant dispersion (including M 22, M 15, M 92 and M 4 Marino et al. 2009; Sobeck et al. 2011; Roederer \& Sneden 2011; Shingles et al. 2014). Last but not least, some color indices may suggest some He enhancement (Milone et al. 2015). Correlation or anticorrelation between those elements have been observed and provide hints to help decipher their origins. While there is a consensus on a hot H-burning nucleosynthesis source, a broad range of polluters have been proposed to explain those chemical trends: fast-rotating massive stars, massive asymptotic giant branch (AGB) stars, intermediatemass binaries, and supermassive stars (see discussion of the various models by Renzini et al. 2015; Charbonnel 2016).

Nevertheless, all those models try to establish a universal scenario for the formation of GCs. One way to distinguish those scenarios may come from confronting the various abundance trends and spread against global properties of the clusters, as already observationally attempted by Carretta et al. (2009a) and Milone et al. (2017) and theoretically predicted by Dell'Agli et al. (2018) and Szécsi \& Wünsch (2019). To do this, large samples of homogeneously analyzed stars are required to allow us to compare trends or (anti)correlations against cluster properties and thus draw an overall picture of GC formation. But, as emphasized by Bastian \& Lardo (2018), to date there have only been a few stars in a handful of GCs that have been fully characterized in terms of their chemistry.

In fact, the largest published spectroscopic homogeneous analysis of GCs stars to date is from Carretta et al. (2009b) who gathered nearly 1400 stellar spectra from the Very Large Telescope/GIRAFFE spectrograph, but these authors study only $\mathrm{Na}$ and $\mathrm{O}$ because of relatively limited resolution. In parallel, Carretta et al. (2009a) measured several elements over 17 GCs, but the sample is only of 202 stars. Given the potential scientific impact of such studies, large spectroscopic surveys have now dedicated generous amount of telescope time for the observation of GCs. Pancino et al. (2017) made use of the Gaia-ESO survey (Gilmore et al. 2012) and studied 510 stars over 9 clusters, but limited their conclusions to $\mathrm{Mg}$ and $\mathrm{Al}$ elements. Mészáros et al. (2015) presented an independent analysis of 428 northern cluster stars using spectra published in the 10th data release of the SDSS III-Apache Point Observatory Galactic Evolution Exper- iment (APOGEE; Ahn et al. 2014). However, this analysis suffered from larger than expected uncertainties in the fundamental $\mathrm{C}$ and $\mathrm{N}$ abundances. Nowadays, the 14th data release (DR14) of the SDSS IV/APOGEE2 survey (Gunn et al. 2006; Zasowski et al. 2017; Majewski et al. 2017; Blanton et al. 2017) contains one of the largest samples of GCs stars with high enough resolution and signal-to-noise ratio to allow the determination of abundances for many key elemental abundances in GCs studies. However, the extreme elemental abundances of GC stars are still uncertain in DR14 standard analysis as demonstrated by Jönsson et al. (2018).

Given the larger than expected errors in the derivation of $\mathrm{C}$ and $\mathrm{N}$ abundances by Mészáros et al. (2015) and the APOGEE Stellar Parameters and Chemical Abundances Pipeline (ASPCAP; Holtzman et al. 2018), we independently revisited the analysis of APOGEE spectra from ten northern GCs and nearly doubled the sample of Mészáros et al. (2015) to 885 because more stars have been observed by APOGEE since then. The current analysis includes measurement of $\mathrm{C}, \mathrm{N}, \mathrm{O}, \mathrm{Mg}, \mathrm{Al}, \mathrm{Si}, \mathrm{K}$, and $\mathrm{Ca}$ as well as the neutron-capture elements $\mathrm{Ce}$ and $\mathrm{Nd}$ for the first time in GCs since those elements have been shown to be measurable in the APOGEE spectra (Hasselquist et al. 2016; Cunha et al. 2017).

\section{Data}

The general steps of target selection were the same as used by Mészáros et al. (2015). After selecting radial velocity members, we cut stars that were beyond the tidal radius given by Harris (2010), and stars that had an offset larger than \pm 0.4 dex from the average ASPCAP DR14 metallicity. Gaia DR2 data have not been used for the member selection because distances are currently not accurate enough for most of these clusters. The new member selection contained 885 stars, which is more than twice as many as the Mészáros et al. (2015) sample of 428 ; most of these new stars were observed after DR10 was published.

The corresponding spectra used in the present work were obtained from the SDSS IV/APOGEE2 survey DR14 (Abolfathi et al. 2018). The spectra have been reduced and combined following Holtzman et al. (2015, 2018), but the spectral normalization has been carried out within our own code.

\section{Analysis}

We use the Brussels Automatic Code for Characterizing High accUracy Spectra (BACCHUS; Masseron et al. 2016) to determine metallicity, microturbulence, and macroturbulence $/ v \sin i$ as well as abundances $(\mathrm{C}, \mathrm{N}, \mathrm{O}, \mathrm{Mg}, \mathrm{Al}, \mathrm{Na}, \mathrm{Ca}, \mathrm{Si}, \mathrm{K}, \mathrm{Nd}$, and $\mathrm{Ce})$. The code runs the spectral synthesis code Turbospectrum (Plez 2012) on the fly and relies on a very large grid of MARCS model atmospheres (Gustafsson et al. 2008), extended over various $\mathrm{C}$ values $(-1.0<[\mathrm{C} / \mathrm{Fe}]<+1.0)$ in addition to more standard parameters $\left(T_{\text {eff }}, \log g\right.$, metallicity). We note that although the grid also contains a range of $[\alpha / \mathrm{Fe}]$ ( $\alpha$ being $\mathrm{O}, \mathrm{Ne}, \mathrm{Mg}$, $\mathrm{Si}, \mathrm{S}, \mathrm{Ar}, \mathrm{Ca}$, and $\mathrm{Ti}$ ), it does not contain models with variation in $\mathrm{O}, \mathrm{Mg}$, and $\mathrm{Si}$, but fixed $\mathrm{Ne}, \mathrm{Ar}, \mathrm{Ca}$, and $\mathrm{Ti}$. Therefore we decided to limit the grid such that $[\alpha / \mathrm{Fe}]=+0.4$. We also stress that the atmospheric models we use do not take into account the variations in $\mathrm{Na}$ or $\mathrm{Al}$, nor possibly in $\mathrm{He}$. The atomic linelist used is from DR14 (see Shetrone et al. 2015, for a description of the linelist building) complemented with $\mathrm{Nd}$ and Ce from (Hasselquist et al. 2016) and Cunha et al. (2017). 


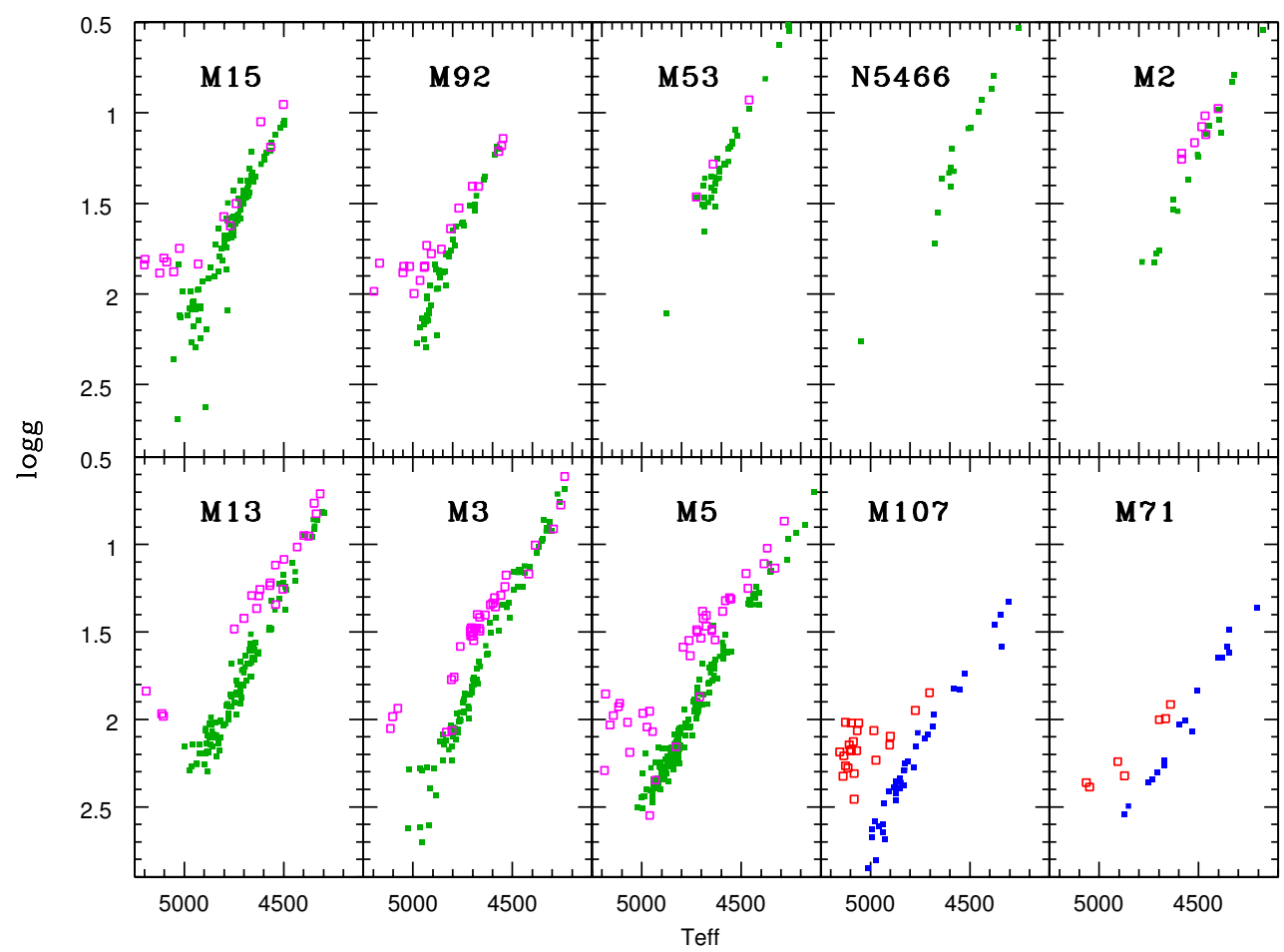

Fig. 1. Effective temperatures and surface gravities derived by the Cannon (DR14) results for sample stars. Open magenta squares indicate RHB/eAGB stars and green full squares indicate RGB stars as determined from photometry except for M 107 and M 71, for which the separation has been done from spectroscopic parameters only. The figure illustrates the potentiality of separating RGB and RHB/eAGB solely from the Cannon results.
The molecular linelists include recent updates from Sneden et al. (2014, CN), Li et al. (2015), Gordon et al. (2017, CO) and Brooke et al. (2016, OH).

The sequence of the spectral analysis procedure is the following: After having fixed the effective temperatures and surface gravities (see Sect. 3.1), the code determines the macroturbulence based on a selection of Si I lines (simultaneously to adjusting the $\mathrm{Si}$ abundance). Then, the metallicity is determined from Fe I lines with macro- and microturbulence parameters fixed. Because of the importance of molecular lines in the $H$ band, we successively determined the $\mathrm{O}, \mathrm{C}$, and $\mathrm{N}$ abundances that dominate the molecular line strengths. Finally, we derived the other element abundances. The overall process was iterated twice to ensure self-consistency and was run over a couple of days for the whole sample on our HTCondor system.

\subsection{Effective temperature and surface gravity}

Jönsson et al. (2018) illustrated that the ASPCAP DR14 analysis leads to an excellent precision in abundances for disk stars and first generation GC stars, lending support for the associated values of effective temperature and gravity. Nevertheless, Jönsson et al. (2018) have also demonstrated that ASPCAP DR14 analyses do not provide satisfying results for second generation GC stars. Moreover, as detailed in Sect. 3.4, we found that the temperatures provided by ASPCAP for GC stars introduce a bias in the oxygen abundances. Our BACCHUS analysis does not determine $T_{\text {eff }}$ and $\log$, so we derived those parameters following Mészáros et al. (2015), i.e., temperatures are inferred from photometry and surface gravities from isochrones. We computed the effective temperatures using the $J-K s$ relation from González Hernández \& Bonifacio (2009), while the reddening values were selected from the Harris catalog (Harris 2010) and kept as constant for each cluster.

In order to assign correctly the surface gravities with isochrones, a distinction between early asymptotic giant branch (eAGB) or red horizontal branch (RHB), and red giant branch
(RGB) stars must be made. This has been done via colorcolor diagrams based on the most accurate ground-based photometry currently available following the same method as García-Hernández et al. (2015). However, such photometric data are only available for the most metal-poor clusters and not for the two most metal-rich clusters in our sample, M 71 and M 107. For these two clusters, we chose to use the Cannon (Ness et al. 2015) analysis of DR14 parameters.

While the accuracy of this machine learning algorithm is limited to that of its training set, it has the advantage of exploiting all of the information that may be present in the stellar spectra, thus improving the precision of the stellar parameters determination. We show in Fig. 1 that the parameters derived by the Cannon allow us to disentangle efficiently the RGB from the RHB/eAGB stars. In comparison, we show in Fig. 2 a similar diagram but with the ASPCAP DR14 calibrated parameters. Although the ASPCAP results also allow us to separate the two giant branches, the Cannon results show this more clearly. Consequently, we separate empirically the RHB/eAGB and the RGB stars in M 107 and M 71 using the Cannon results. This provides a new method for separating the evolutionary status of giants in clusters, or in monoage populations in general. In contrast, we found that the effective temperatures solely from $H$-band spectroscopy; the surface gravities provided by the Cannon are much less accurate than the ASPCAP values, thus the latter are preferred for parameter determinations of GCs. We note that a few stars in M 5, M 13, M 3, and M 15 have been apparently misclassified by the Cannon and/or ASPCAP. However, it is not clear at this stage of this study whether it is due to an error in the Cannon determination of the parameters or an error in the photometric classification. More work would be needed to clarify the situation of those few outliers and is beyond the scope of this paper.

Once the evolutionary status is assigned, the surface gravities are determined by using the set of isochrones from the Padova group (Bertelli et al. 2008; Marigo et al. 2017). We use 12 billion years isochrones for all GCs, except for M 107 and M 71 for which we use 10 billion years. Regarding metallicities, 


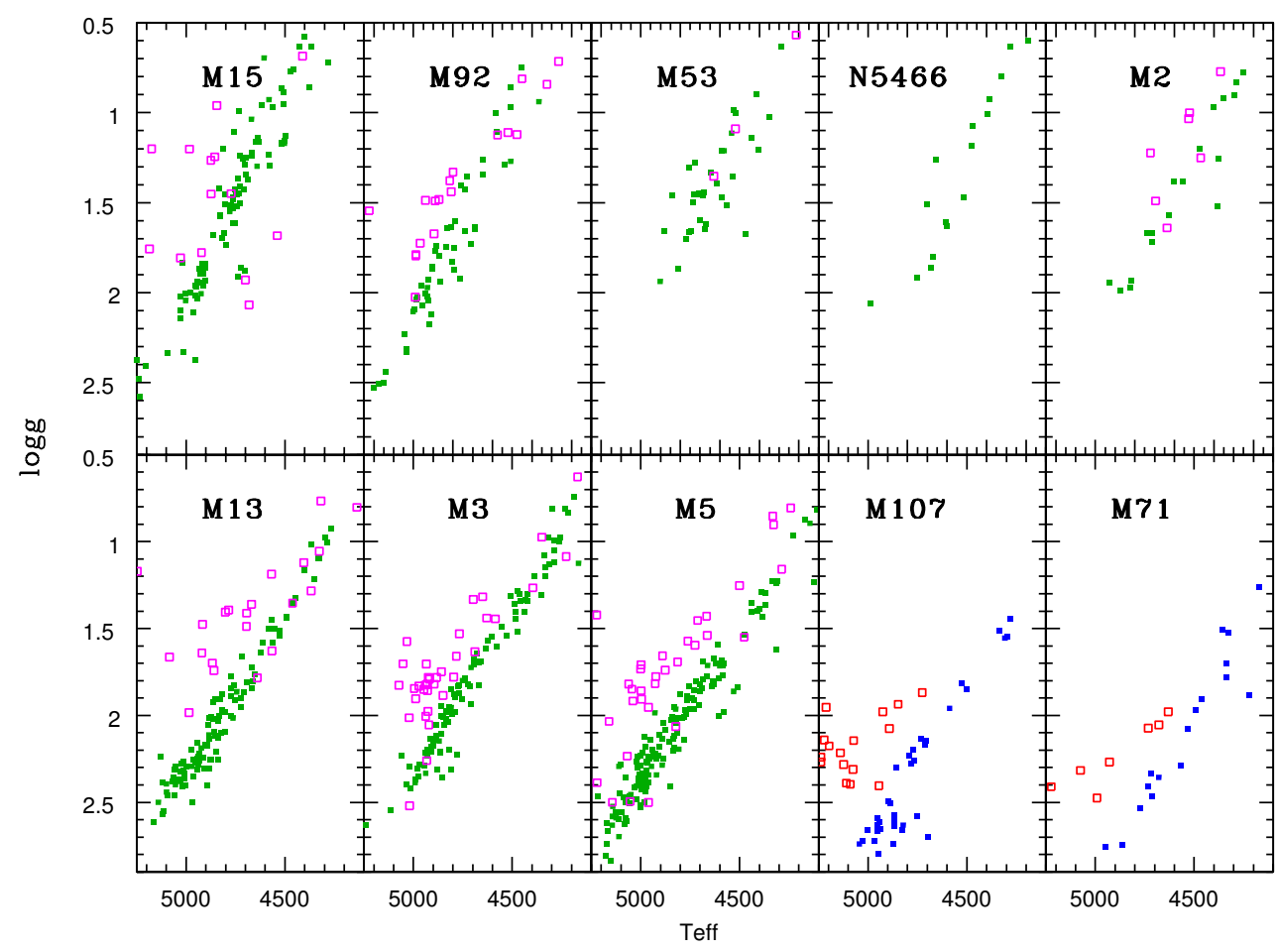

Fig. 2. Effective temperatures and surface gravities derived by ASPCAP (DR14) for the sample stars. Open magenta squares indicate RHB/eAGB stars and green filled squares indicate RGB stars as determined from photometry except for M107 and M71, for which the separation has been done from spectroscopic parameters only. In comparison to Fig. 1, this figure illustrates that the Cannon work has more precise results when it comes to separating the RGB/RHB/eAGB, but as shown in Jönsson et al. (2018), the ASPCAP parameters are more accurate when compared to independently analyzed local disk stars. we adapt the isochrones to the metallicities of the Harris (2010) catalog.

\subsection{Micro- and macroturbulence}

Microturbulence velocities have been fixed to the relation obtained in the optical by the Gaia-ESO survey (such as described by Smiljanic et al. 2014). However, in order to check the validity of this relation in the $H$-band infrared spectra of APOGEE, we let the microturbulence parameter free in a preliminary run. The code determines the microturbulence by cancelling the trend of abundances against equivalent widths of a set of Fe I lines. Figure 3 shows the results obtained with BACCHUS and compares these to the values derived in APOGEE DR14 and to the relation of the Gaia-ESO survey. All the relations agree well and show in particular an expected dependence with surface gravity. Nevertheless, the dispersion of the microturbulence values increase significantly for the most metal-poor stars $([\mathrm{Fe} / \mathrm{H}]<-1.5)$. This is because the lines used for the microturbulence relation become very weak and very sensitive to random uncertainties such as noise or continuum placement, and thus increase the uncertainties of the microturbulence relation. Because our study contains a large number of very lowmetallicity stars, we decided to fix the microturbulence velocity to the optical relation from Gaia-ESO to reduce the impact of the growing error at low metallicity on the abundances.

The BACCHUS code does not derive the macroturbulence value directly, but rather derives the value of the spectral convolution necessary to match the observed line profile after the thermal, radiative, collisional, and microturbulence broadening are taken into account in the synthesis. This convolution parameter approximately represents the quadratic sum of instrumental resolution, projected velocity of the $\operatorname{star}(v \sin i)$, and macroturbulence. To determine this value, the BACCHUS code iterates over a series of selected lines until it finds convergence between abundances measured from the few points around the core of the line (being sensitive to the convolution value) and from the

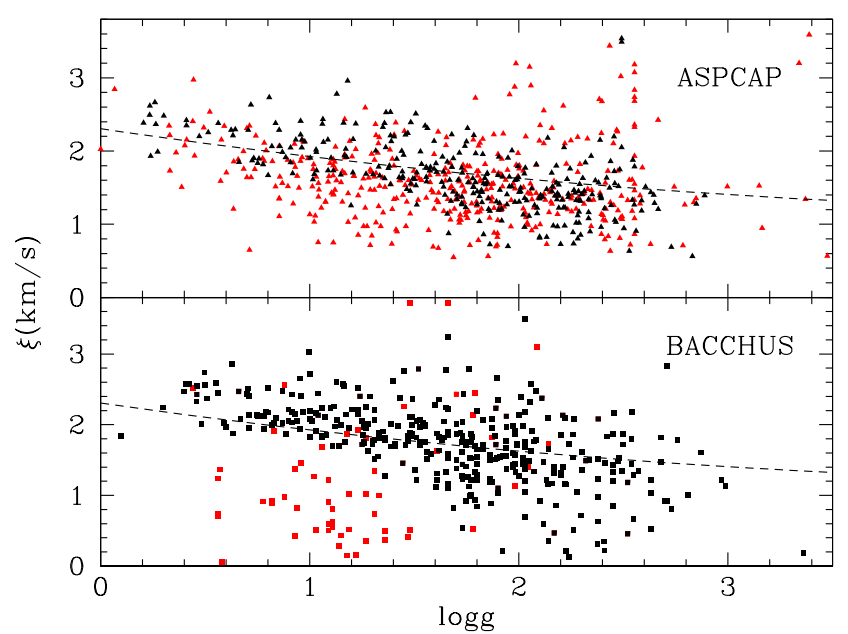

Fig. 3. Microturbulence velocity values obtained for the sample stars by the ASPCAP pipeline (upper panel) and by our preliminary run with the BACCHUS code (lower panel). Red points indicate stars with $[\mathrm{Fe} / \mathrm{H}]<-1.5$ and black points stars with $[\mathrm{Fe} / \mathrm{H}]>-1.5$. The dashed line shows the relation derived in the optical by the Gaia-ESO survey assuming $T_{\text {eff }}=4500 \mathrm{~K}$ and $[\mathrm{Fe} / \mathrm{H}]=-1.5$. Both works show a good correspondence with the optical data for the metal-rich stars, but a larger dispersion is obtained for low-metallicity stars.

equivalent width of the lines (insensitive to the convolution). For this particular analysis, we used a Gaussian kernel for the convolution and probe it over a set of clean Si I lines; those lines being strong enough in all our metal-poor stars in contrast to Fe I lines (see next Subsection).

In Fig. 4, we show the resulting convolution values derived from our procedure against $\log g$ and metallicity. We highlight the average fiber number of each target in the figure. It is remarkable that we obtain higher convolution values on average for stars with a larger fiber number than those with a lower fiber number. This is fully consistent with the fact that the APOGEE 

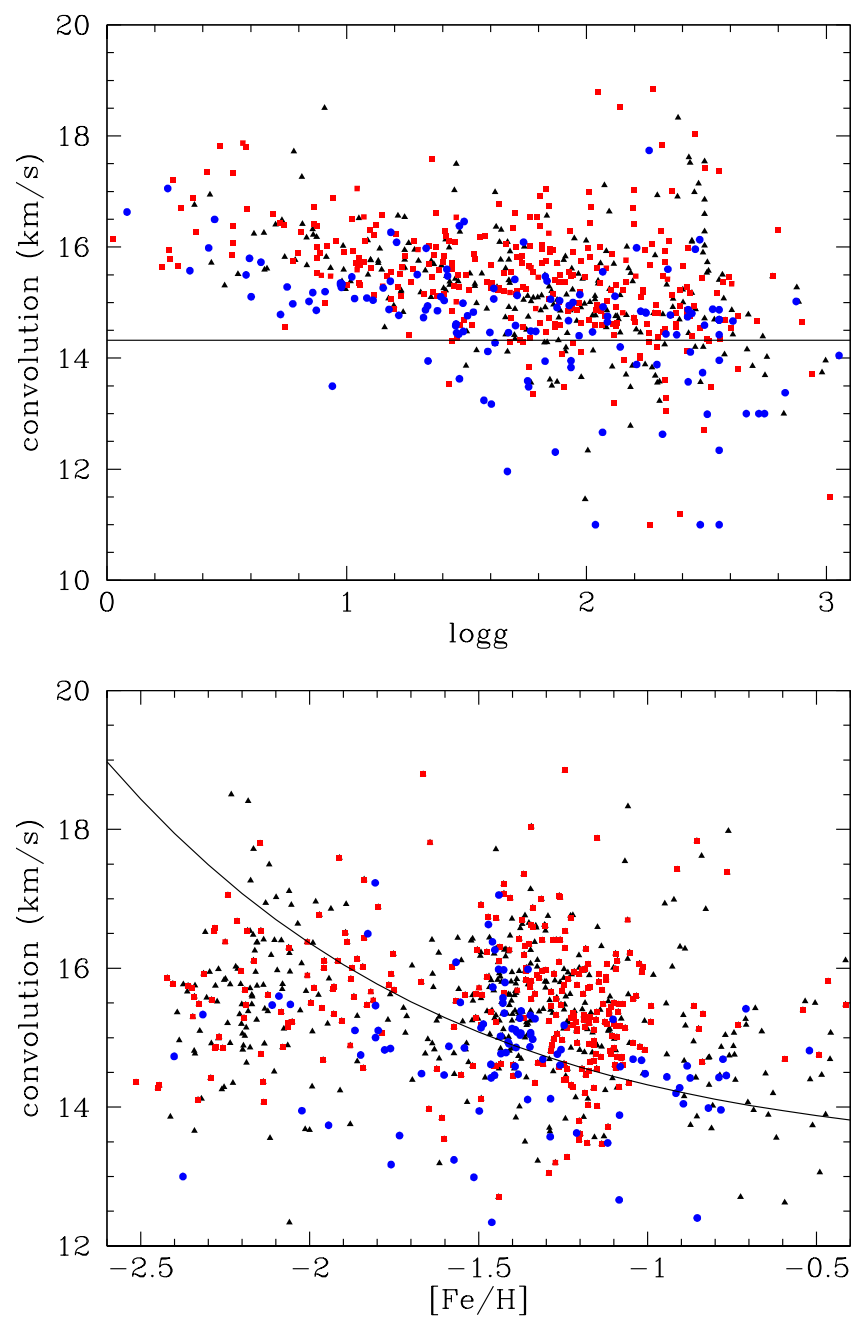

Fig. 4. Convolution values obtained for the sample stars against surface gravities (upper panel) and metallicity (lower panel). Red squares indicate spectra with a low mean fiber number $(<100)$, blue points indicate spectra with a high mean fiber number $(>200)$, and black triangles indicate the others. The continuous line corresponds to the macroturbulence relation adopted in APOGEE DR14 quadratically summed with an average resolution of $13.3 \mathrm{~km} \mathrm{~s}^{-1}(R \sim 22500)$.

spectrograph has a variable resolution depending on the fiber (Nidever et al. 2015). Although the BACCHUS code makes some approximations regarding the macroturbulence and instrument line profile broadening, the recovery of the fiber impact on the spectra demonstrates the ability of the code to account for the various sources of line broadening parameters when deriving the stars abundances.

In the same Figure, we compare our convolution values to the adopted value for macroturbulence from DR14 APOGEE data release (Holtzman et al. 2018). Holtzman et al. (2018) have found a dependence only in metallicity in DR14. Our results tend to rather show a dependence in $\log g$, which is consistent with previous findings from Hekker \& Meléndez (2007), Gray (2008), but we do not find a dependence on metallicity. The macroturbulence velocity relation derived in Holtzman et al. (2018) is based on the whole APOGEE sample, which is dominated by more metal-rich stars than present in our GC sample and may be responsible for some of the disagreement. In any case, we stress that if the macroturbulence is really overestimated at low metallicity, we expect that all abundances and parameters derived from the ASPCAP

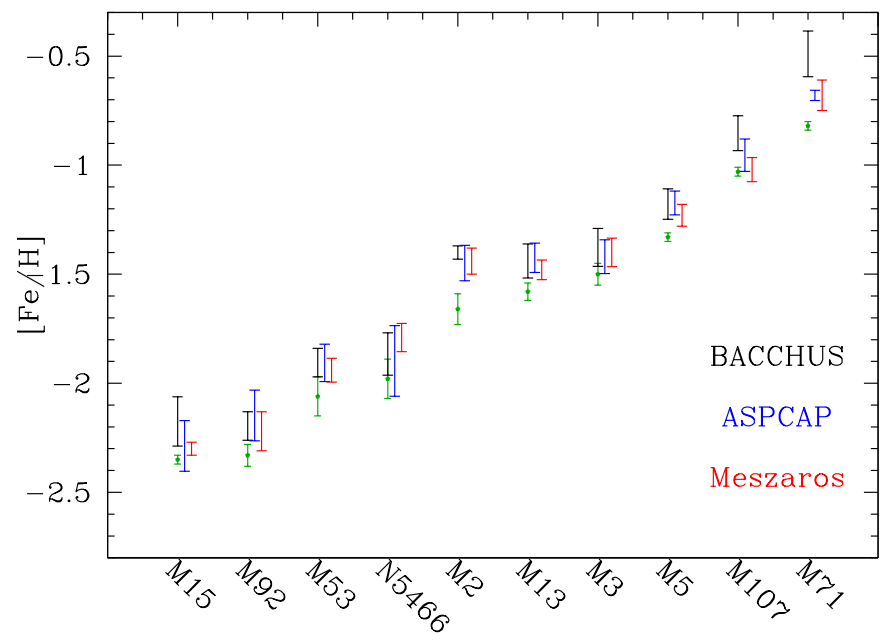

Fig. 5. Median metallicities obtained by the present work, APOGEE DR14, and Mészáros et al. (2015) for the 10 same GCs stars. Error bars indicate the star-by-star metallicity rms. Green points indicate literature values (Carretta et al. 2009c).

pipeline to be significantly affected for all stars with metallicities below -2.0 .

\subsection{Metallicity}

Once the macroturbulence, microturbulence, $T_{\text {eff }}$, and $\log g$ have been fixed, the metallicity is derived from a selection of three Fe I lines. Fig. 5 shows the median metallicity obtained for each cluster and compared to various literature values. The agreement between all the studies is good overall. While there is evidence for metallicity variations within some peculiar clusters (e.g., $\omega$ Cen, NGC 6934, Norris \& Da Costa 1995; Marino et al. 2018), the relatively low star-to-star metallicity dispersion as shown in Fig. 5 is consistent with the conclusions of Carretta et al. (2009c), claiming that most of the clusters can be considered mono-metallic regarding the Fe abundance. However, we find a systematic offset of $\sim 0.1$ dex in metallicity compared to the optical values for all clusters, as do other studies of the APOGEE spectra (Mészáros et al. 2015; Holtzman et al. 2018). Mucciarelli et al. (2015b) already demonstrated that non-local thermodynamic equilibrium (NLTE) effects in optical Fe I lines in M 2 stars affect the metallicity. In the $H$ band we also strongly suspect 3D-NLTE effects, notably because 1DLTE synthesis of a very high resolution of Arcturus spectrum does not provide a satisfying fit for Fe I line profiles. A more extended discussion will be given in a forthcoming paper. It is for this reason that we carefully select only three Fe I lines that are as insensitive as possible to NLTE effects to determine the metallicities. In any case, such an offset in metallicity does not affect our discussion because we mostly focus on the relative trends or ranges of abundance-abundance diagrams, cluster by cluster.

\subsection{Abundances}

The abundances have been derived by the code on a line-by-line basis. The lines used for abundance determination are shown in Table 1 . We note that the BACCHUS code automatically adjusts the window/mask on a star-by-star and line-by-line basis (see the manual for more details Masseron et al. 2016). Therefore we provide only central wavelengths in Table 1. Among 
Table 1. Lines used in this work for abundance determination.

\begin{tabular}{ll}
\hline \hline Element & Wavelength $\left(\AA_{\text {air }}\right)$ \\
\hline $\mathrm{C}(\mathrm{CO})$ & 15578.015775 .515978 .716185 .516397 .216614 .0 \\
& 16836.017063 .017448 .617456 .0 \\
$\mathrm{~N}(\mathrm{CN})$ & 15119.015210 .215222 .015228 .815242 .515251 .8 \\
& 15309.015317 .615363 .515410 .515447 .015462 .4 \\
& 15466.215495 .015514 .015581 .015636 .515659 .0 \\
& 15825.715391 .015569 .015778 .516052 .916055 .5 \\
& 16650.016704 .816714 .516872 .016909 .4 \\
$\mathrm{O}(\mathrm{OH})$ & 15391.015569 .015778 .516052 .916055 .516650 .0 \\
& 16704.816714 .516872 .016909 .4 \\
$\mathrm{Na} \mathrm{I}$ & 16373.916388 .8 \\
$\mathrm{Mg} \mathrm{I}$ & 15740.715749 .015765 .7 \\
$\mathrm{Al} \mathrm{I}$ & 16719.016750 .6 \\
$\mathrm{Si} \mathrm{I}$ & 15361.215376 .815557 .815827 .215833 .615884 .5 \\
& 15960.116060 .016094 .816129 .016163 .716170 .2 \\
$\mathrm{~K} \mathrm{I}$ & 16215.716241 .816680 .816828 .2 \\
$\mathrm{Ca}$ I & 15163.015168 .3 \\
$\mathrm{Fe}$ I & 16136.816150 .816155 .216157 .416197 .1 \\
$\mathrm{Ce}$ II & 15277.615784 .715829 .815958 .415977 .116327 .3 \\
$\mathrm{Nd} \mathrm{II}$ & 16376.416595 .116522 .5 \\
& 15284.415368 .115912 .215977 .916053 .616262 .0 \\
& 16303.716382 .916558 .216634 .6 \\
\hline
\end{tabular}

the several abundance indicators that the code offers (equivalent width, core line intensity or $\chi^{2}$ ) and their respective flags, we selected the abundances corresponding to the minimum $\chi^{2}$, but still use the other methods to reject any suspicious line. The final abundance is the mean of the abundances of the nonflagged lines.

\subsubsection{Errors}

Systematic errors have been evaluated by comparing the abundances obtained using the uncalibrated effective temperature and surface gravities from APOGEE DR14 and running the BACCHUS code following the same procedure as described above. The corresponding results are reported for each star of the sample in Fig. 6 and compared to the mean random uncertainties (the latter being derived from the line-by-line abundance dispersion). In this figure, systematic uncertainties are comparable to random uncertainties for most of the elements. However, these uncertainties appear significantly larger for $\mathrm{C}, \mathrm{N}, \mathrm{O}, \mathrm{Nd}$, and Ce. This is because of the high sensitivity of their abundances to effective temperature and/or surface gravity. Indeed, the APOGEE DR14 parameters tend to show systematic parameter differences with optical spectroscopy, as highlighted by Jönsson et al. (2018), affecting the abundance determination of the most sensitive elements. Nevertheless, in Fig. 6 we illustrate the impact of systematic errors on the abundances of $\mathrm{Na}, \mathrm{Mg}$, $\mathrm{K}, \mathrm{Si}, \mathrm{Ca}$, and $\mathrm{Fe}$ as well as on $\mathrm{C}, \mathrm{N}$, and $\mathrm{O}$, by using another set of effective temperature or surface gravity. These effects are relatively small compared to the intrinsic variations observed in GCs (see Sect. 4), and thus do not affect our conclusions regarding those elements.

\subsubsection{Upper limits}

Determining upper limits of abundances can be particularly useful when lines become too weak to be accurately measured.

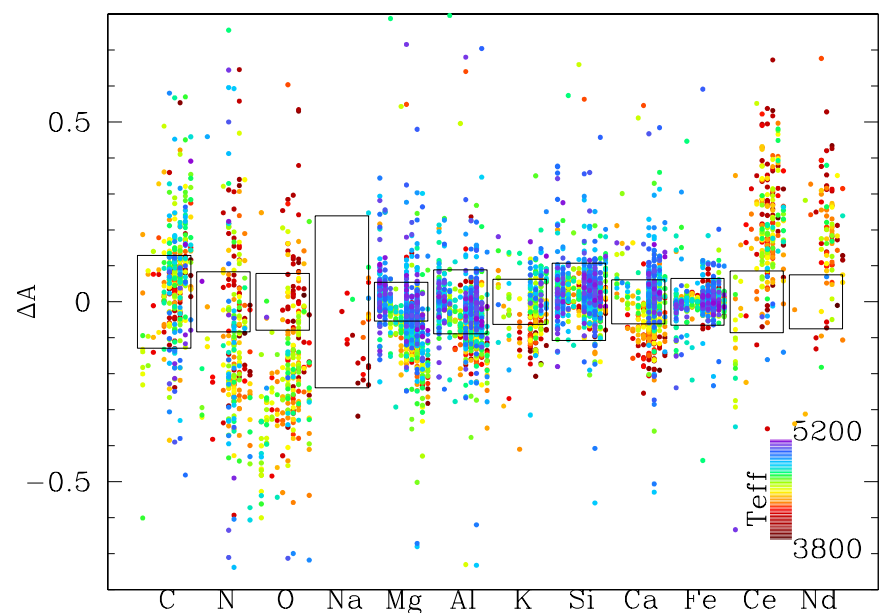

Fig. 6. Difference in abundances obtained by BACCHUS using APOGEE $T_{\text {eff }}$ and $\log g$ with those obtained using our parameters. The points are color-coded by temperature and ordered by cluster metallicity for each element. The squares represent the mean random line-by-line scatter uncertainties of each element. Systematic uncertainties are comparable to random uncertainties except for $\mathrm{C}, \mathrm{N}, \mathrm{O}, \mathrm{Ce}$, and $\mathrm{Nd}$.

Actually, many lines used for abundance determination become particularly weak at the metallicity of many GC spectra. The BACCHUS code is able to determine for each line an upper limit to abundances by comparing the variance of the observed spectrum to the behavior of the line strength in the synthetic spectra. The upper limit corresponds to the abundance where the line intensity is comparable to the variance, and is consequently in BACCHUS sensitive to stellar parameters, as well as spectral resolution or signal-to-noise ratio. An illustration of the importance of flagging and determining upper limits is given in Fig. 7. In this figure, we can observe that most of the upper limits for $\mathrm{C}$, $\mathrm{N}$, and $\mathrm{O}$ elements are set for stars with effective temperatures above $4600 \mathrm{~K}$ (100 K higher than what Mészáros et al. (2015) used). This naturally occurs because the strength of the molecular lines used for those elements quickly weakens as temperature increases. However, while a flagging system is clearly missing in the ASPCAP pipeline (García Pérez et al. 2016), and it is unclear to which extent the pipeline can actually measure those elements. Furthermore, to avoid any bias in the interpretation and discussion of our results, we do not show $\mathrm{C}, \mathrm{N}$, and $\mathrm{O}$ abundances for stars with $T_{\text {eff }}>4600 \mathrm{~K}$.

\subsubsection{Comparison with literature}

Figures 7-9 show the resulting abundances as functions of effective temperature for three distinct analysis of the same APOGEE spectra: this work, the uncalibrated ASPCAP DR14 results, and those from Mészáros et al. (2015). For clarity, we do not show the entire sample in these diagrams, but rather select the most relevant stars or clusters to highlight any residual trends or offsets.

Apart from the stars with upper limits, the abundances we derive agree fairly well with previous studies. Nevertheless, in Fig. 7 we notice that the $\mathrm{N}$ values derived by the ASPCAP pipeline for the DR14 data do not reach values higher than 1.0. This is issue is due to the limits of the model grid, which will be enlarged for the next 16th data release of SDSS. Nevertheless, we note that such extreme values in $\mathrm{N}$ and $\mathrm{C}$ are only expected in specific cases such as GCs stars (e.g., Mészáros et al. 2015) or peculiar N-rich stars (Fernández-Trincado et al. 2016, 2017, 2018; Schiavon et al. 2017). Mészáros et al. (2015) extended the 


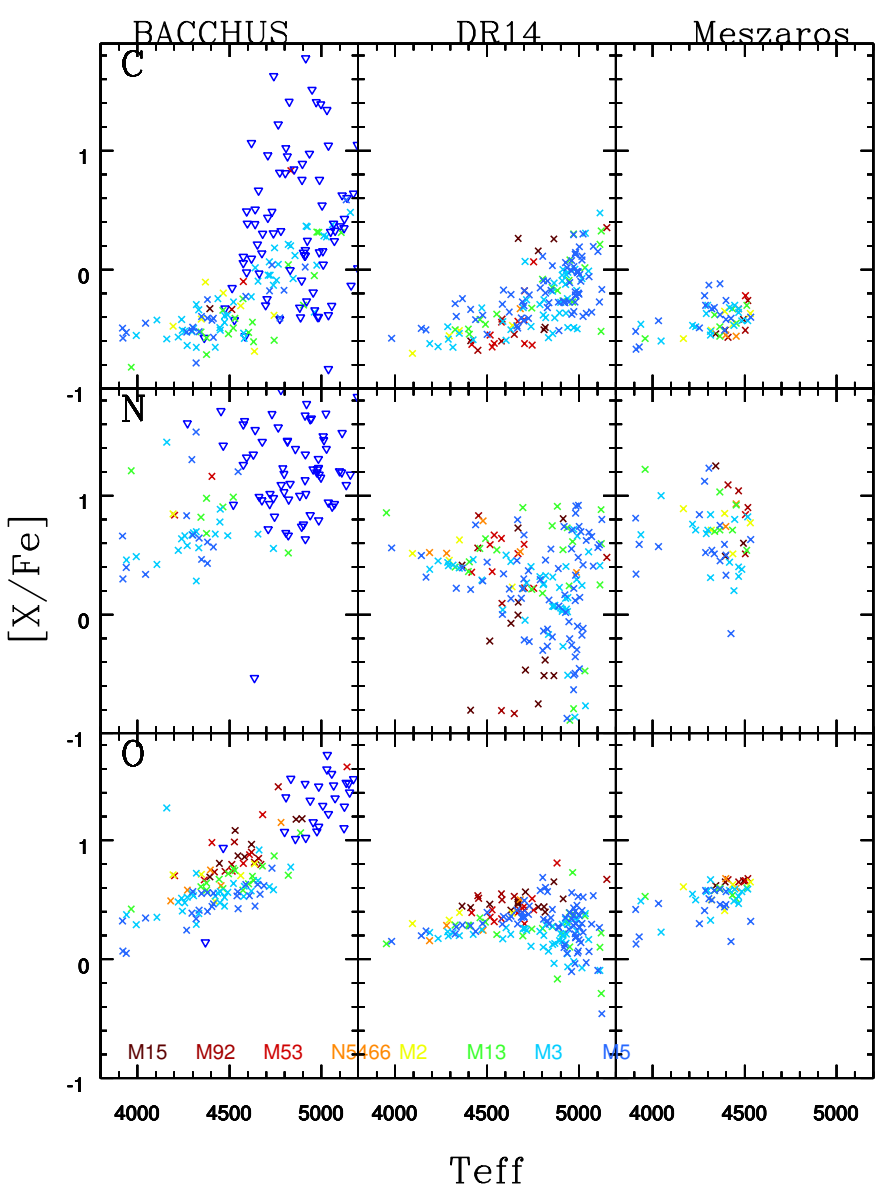

Fig. 7. $\mathrm{C}, \mathrm{N}$, and $\mathrm{O}$ abundances as functions of effective temperature for the same stars as functions of effective temperature for three studies (this work, first column; uncalibrated APOGEE DR14, second column; and Mészáros et al. 2015, third column). Blue triangles indicate upper limits. Our results show that $\mathrm{C}, \mathrm{N}$, and $\mathrm{O}$ abundances have mostly upper limits for $T_{\text {eff }}>4600 \mathrm{~K}$, whereas the APOGEE DR14 results do not provide upper limits. Mészáros et al. (2015) chose not to provide $\mathrm{C}, \mathrm{N}, \mathrm{O}$ values for $T_{\text {eff }}>4500 \mathrm{~K}$. There is a significant trend in our $\mathrm{O}$ determinations compared to the two other studies for which we do not have a clear explanation (see text).

limit of $[\mathrm{N} / \mathrm{Fe}]$ up to +1.5 , but still restrict $[\mathrm{C} / \mathrm{Fe}] \geq-0.75$, which made the abundances of the most $\mathrm{C}$-poor and N-rich stars unreliable.

Furthermore, none of the measured elements seem to show a temperature dependence consistent with the results in literature, except for $\mathrm{C}, \mathrm{N}$, and $\mathrm{O}$. While the $\mathrm{C}$ trend is probably related to expected changes in the evolution of giants, $\mathrm{N}$ should rather increase with decreasing temperatures while $\mathrm{O}$ is not expected to remain constant with temperature. Moreover, both the Mészáros et al. (2015) and APOGEE DR14 results show a slight trend in [O/Fe] as a function of $T_{\text {eff }}$, but our is significantly stronger. The following hypotheses for such a trend can be invoked:

- Our chosen $T_{\text {eff }}$-color transformation may be poorly calibrated for metal-poor GCs stars (González Hernández \& Bonifacio 2009). Given the extreme sensitivity of O in the $H$ band as demonstrated by Jönsson et al. (2018), the $\mathrm{O}$ trend may be a residual from this color- $T_{\text {eff }}$ approximation. However, our temperature scale is similar to Mészáros et al. (2015), thus should lead to a similar trend; this, however, is not the case

- We used spherical stellar atmospheres, whereas APOGEE DR14 and Mészáros et al. (2015) used plane parallel models.

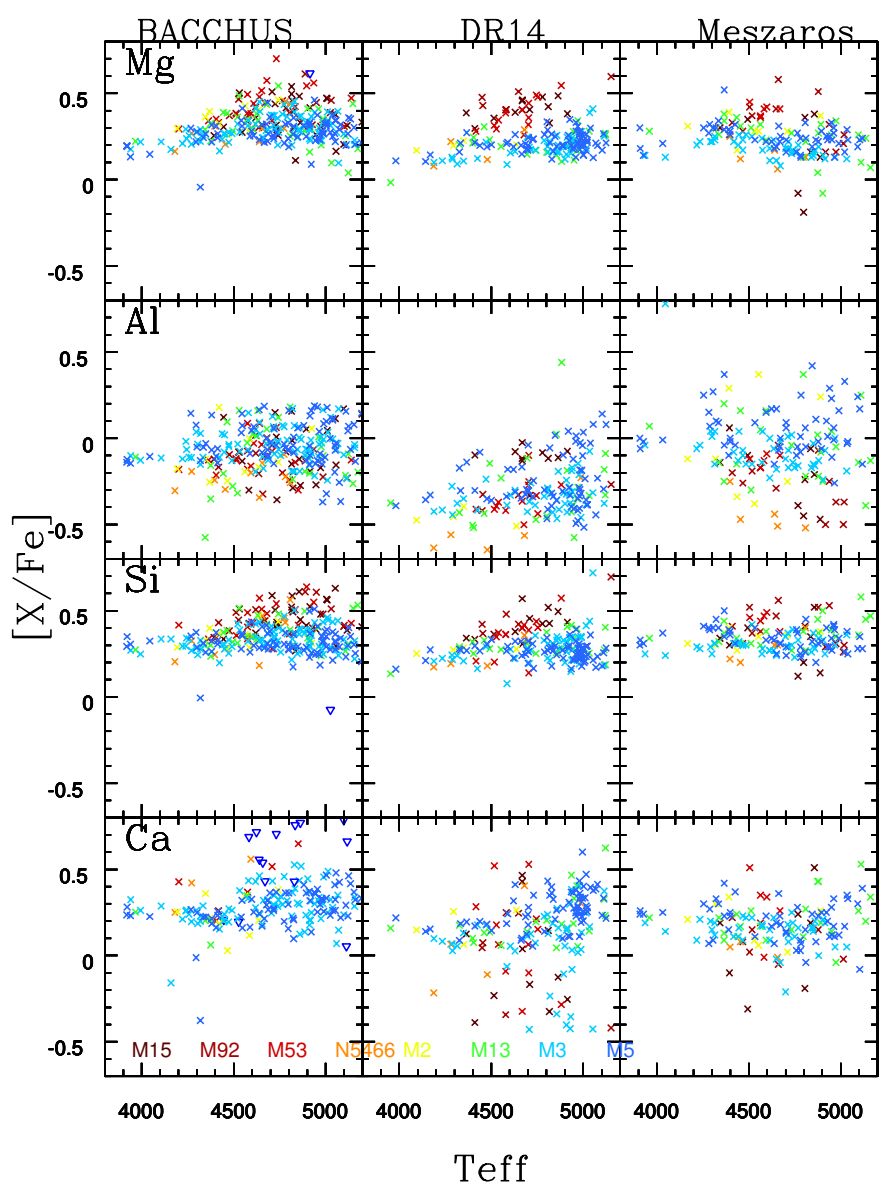

Fig. 8. Comparison $\mathrm{Mg}, \mathrm{Al}, \mathrm{Si}$, and $\mathrm{Ca}$ abundances for the same stars as functions of effective temperature for three studies (this work, first column; uncalibrated APOGEE DR14, second column; and Mészáros et al. 2015, third column). Blue triangles indicate upper limits. To clear up the diagrams with intrinsic clusters abundances variations, only stars of the first population $([\mathrm{Al} / \mathrm{Fe}]<0.2)$ are shown. For clarity sake, the results for two more metal-rich clusters M 71 and M 107 have been disregarded in this figure.

The differences basically lead to a cooler layer in the outer layers in the spherical case compared to the plane parallel, where molecular lines form. Consequently, the $\mathrm{OH}$ molecule from which $\mathrm{O}$ is measured is stronger in the synthesis with spherical models and thus leads to lower $\mathrm{O}$ abundances. While this may explain the difference between our study and previous analysis, the fact that we used an improved approximation for radiative transfer and the model atmosphere still does not explain why we obtain a trend.

- It has been demonstrated that 3D corrections affect the $\mathrm{OH}$ lines in the $H$ band at very low metallicity (Dobrovolskas et al. 2015), such that $O$ abundances are overestimated in a 1D analysis. But this effect is opposite from what is observed, where the coolest giants with larger 3D effects should have even lower $\mathrm{O}$ than shown in Fig. 7.

- Last, we used model atmosphere with $\alpha$ elements composition (including $\mathrm{O}, \mathrm{Mg}$, and $\mathrm{Si}$ ), as well as $\mathrm{Na}$ and $\mathrm{Al}$, fixed. But in GCs, O, Si, and $\mathrm{Mg}$ are known to vary independently. Moreover, $\mathrm{Al}$ and $\mathrm{Na}$ are also known to vary in GCs. Those elements are non-negligible electron donors, which could also affect the atmospheric structure. We tested the impact of changing the initial $\alpha$ elements content by $0.5 \mathrm{dex}$, but we obtained no significant change regarding the trend of $\mathrm{O}$ versus effective temperature. 


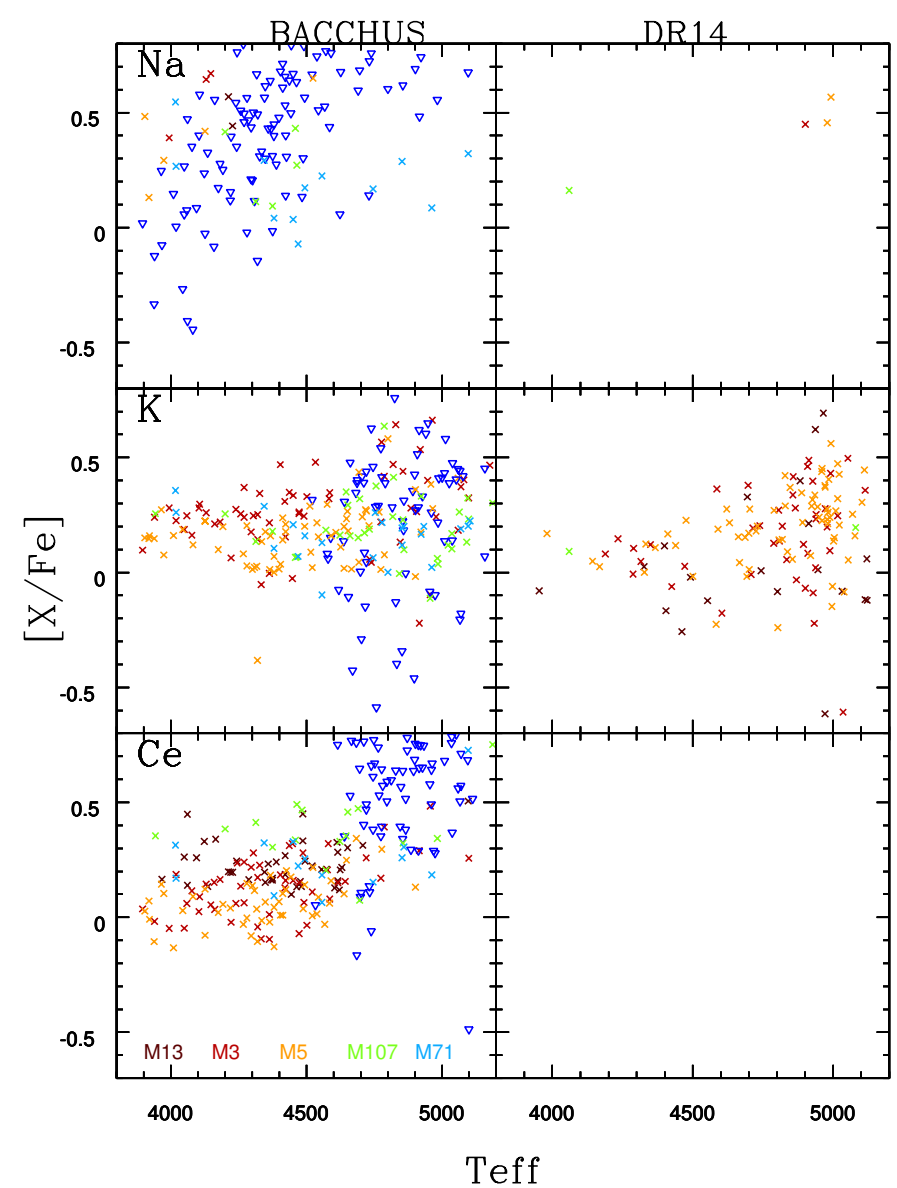

Fig. 9. Comparison $\mathrm{Na}, \mathrm{K}$, and $\mathrm{Ce}$ abundances as functions of effective temperature for two studies (this work, first column and uncalibrated APOGEE DR14, second column). Blue triangles indicate upper limits. The more metal-poor clusters are not shown because those clusters contain mostly upper limits values.

Finally, we derived $\mathrm{O}$ abundances with the BACCHUS code adopting the calibrated temperatures and surface gravities in APOGEE DR14. In Fig. 10 we show these abundances as functions of the APOGEE uncalibrated temperatures and against Al. With the temperatures provided by APOGEE DR14, we can reproduced very well the APOGEE DR14 results and we do not observe anymore any significant trend with $T_{\text {eff }}$ as we do with our photometric temperatures. But those measurements do not exhibit any oxygen variations in clusters and the wellestablished Al-O anticorrelation. This is clearly in contradiction with the findings from previous GCs cluster studies. Knowing that $\mathrm{OH}$ is very temperature sensitive in the $H$ band, we interpret such a paradox to mean that the ASPCAP is biasing the effective temperatures to obtain $\mathrm{O}$ abundances such that the built-in relation $[\mathrm{O} / \alpha]=0$ is satisfied. This interpretation is very well in line with the comparison of ASPCAP results against literature by Jönsson et al. (2018). Therefore, although there may still be some systematic uncertainties leading to a trend in $\mathrm{O}$ against temperature, we conclude that using our photometric temperatures provides more realistic results than with those from ASPCAP DR14 for the study of GCs .

While an increasing trend of $\mathrm{N}$ with decreasing temperature is expected because of internal $\mathrm{CN}$ cycling along the RGB, our data rather seem to show the opposite (middle panel of Fig. 7). Masseron \& Gilmore (2015) demonstrated that CN lines (thus N abundances) are extremely sensitive to the $\mathrm{O}$ abundances. Therefore, it is likely that the trend obtained is related to the $\mathrm{O}$ trend.
Teff

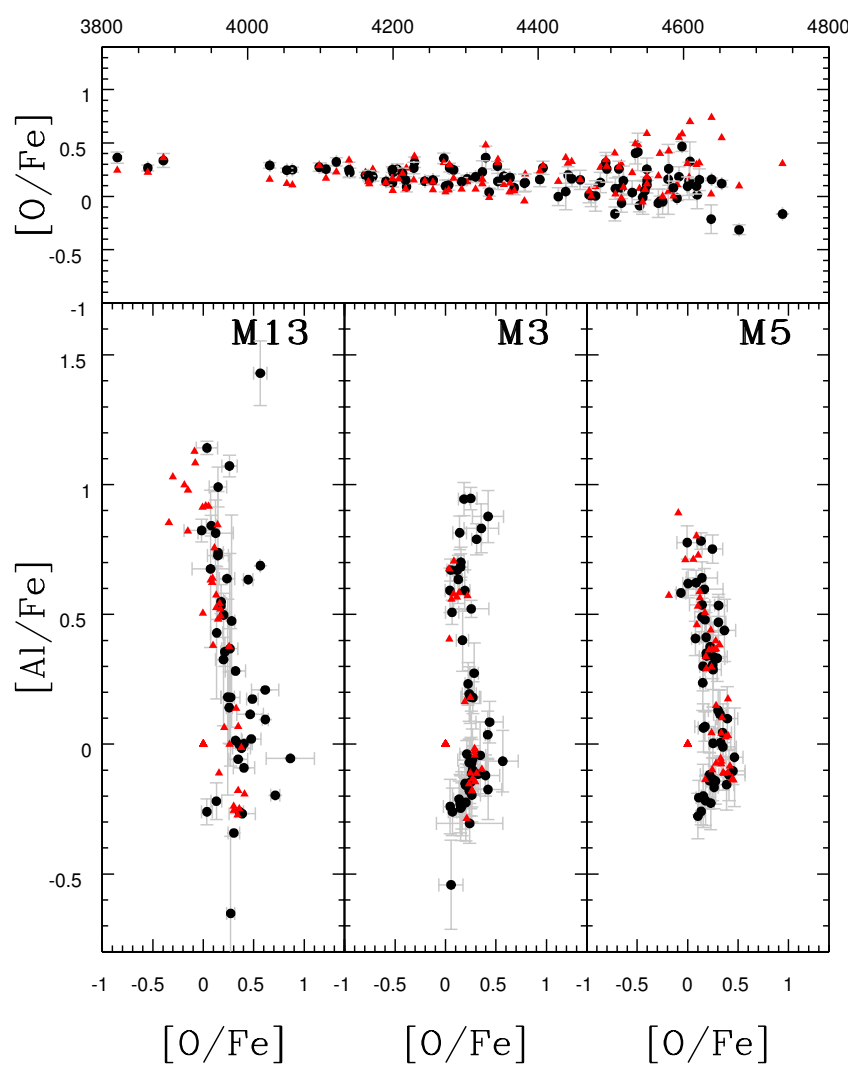

Fig. 10. O abundances as functions of effective temperature (upper panel) and against $\mathrm{Al}$ abundances for three of our clusters (bottom panels) determined by the BACCHUS code with the APOGEE DR14 uncalibrated $T_{\text {eff }}$ and $\log g$ (black dots) and by the ASPCAP pipeline (uncalibrated values, red triangles). The oxygen trend is slightly positive in contrast to Fig. 7. Moreover, almost no $\mathrm{O}$ dispersion nor anticorrelation is observed, which cast some doubts on the stellar parameters.

In any case, whenever this trend in $\mathrm{O}$ is due to a measurement bias or not, there is also still the possibility that this trend is real. Indeed, D'Antona \& Ventura (2007) and Di Criscienzo et al. (2018) predicted that $\mathrm{O}$ depletion may occur along the RGB when stars are enriched in $\mathrm{He}$ as this could be the case in GC stars (Lardo et al. 2018; Milone et al. 2018). Therefore, without a clear understanding of the reason behind the $\mathrm{O}$ trend, we chose not to correct it. Moreover, as shown in Sect. 4, the observed intrinsic spreads in $\mathrm{O}$ and in $\mathrm{N}$ are larger than their amplitudes. Therefore, although this may affect the precision of our abundances it does not affect our conclusions.

\section{Discussion}

Final abundances, number of lines used, upper limits, and random errors are reported in Table 2. For some stars, the Fe I lines were too weak to be detected and only upper limits could be obtained for the metallicity. For those stars, the literature metallicity values from Harris (2010) were adopted to build the plots. All plots in the following section display random errors.

\subsection{Na-O and O-Al anticorrelations}

$\mathrm{Na}$ is very difficult to measure in the APOGEE spectra of metalpoor stars because the $\mathrm{Na}$ I lines are very weak. Actually, those lines appear only in the coolest stars of the most metal-rich 
Table 2. Parameters and abundances for the sample stars.

\begin{tabular}{|c|c|c|c|c|c|c|c|c|c|c|c|c|c|}
\hline Star & Cluster & Status* & $T_{\text {eff }}$ & $\log g$ & {$[\mathrm{Fe} / \mathrm{H}]$} & $\sigma$ & {$[\mathrm{C} / \mathrm{Fe}]$} & $\sigma$ & $\#$ & {$[\mathrm{~N} / \mathrm{Fe}]$} & $\sigma$ & $\#$ & $\cdots$ \\
\hline $2 \mathrm{M} 15184612+0204467$ & M5 & $\mathrm{HB}$ & 7054 & 3.01 & $\cdots$ & $\ldots$ & $\ldots$ & $\ldots$ & 0 & $\cdots$ & $\cdots$ & 0 & $\ldots$ \\
\hline $2 \mathrm{M} 15184730+0207253$ & M5 & eAGB & 4998 & 1.70 & -1.314 & 0.129 & 0.182 & 0.171 & 1 & $<1.279$ & .. & 1 & $\ldots$ \\
\hline $2 \mathrm{M} 15185167+0201178$ & M5 & eAGB & 5061 & 1.81 & -1.358 & 0.046 & $\ldots$ & $\ldots$ & 0 & $\ldots$ & .. & 0 & $\ldots$ \\
\hline $2 \mathrm{M} 15185197+0211217$ & M5 & $\mathrm{HB}$ & 5681 & 2.02 & -1.575 & 0.345 & 1.554 & 0.094 & 1 & $<2.578$ & $\ldots$ & 1 & $\ldots$ \\
\hline $2 \mathrm{M} 15185499+0205525$ & M5 & $\mathrm{HB}$ & 6366 & 2.30 & $\ldots$ & $\ldots$ & $\ldots$ & $\ldots$ & 0 & $\ldots$ & $\ldots$ & 0 & $\ldots$ \\
\hline $2 \mathrm{M} 15185515+0214337$ & M5 & eAGB & 4663 & 1.53 & -1.236 & 0.048 & -0.313 & 0.125 & 1 & $<1.131$ & $\cdots$ & 1 & $\ldots$ \\
\hline $2 \mathrm{M} 15185731+0203077$ & M 5 & eAGB & 4995 & 1.90 & -1.206 & 0.007 & $<1.164$ & $\ldots$ & 1 & $<2.519$ & .. & 1 & $\ldots$ \\
\hline $2 \mathrm{M} 15190831+0201421$ & M5 & $\mathrm{HB}$ & 5394 & 2.04 & -1.206 & 0.121 & $\cdots$ & $\cdots$ & 0 & $\cdots$ & $\cdots$ & 0 & $\cdots$ \\
\hline $2 \mathrm{M} 15191829+0209175$ & M5 & $\mathrm{HB}$ & 5934 & 2.48 & $\ldots$ & $\ldots$ & $\ldots$ & $\ldots$ & 0 & $\ldots$ & $\ldots$ & 0 & $\ldots$ \\
\hline $2 \mathrm{M} 15193344+0205072$ & M 5 & $\mathrm{HB}$ & 7092 & 3.37 & $\cdots$ & $\ldots$ & $\cdots$ & $\ldots$ & 0 & $\ldots$ & $\cdots$ & 0 & $\cdots$ \\
\hline 2M16315197-1303389 & M 107 & RGB & 4950 & 2.66 & -1.071 & 0.065 & -0.326 & 0.279 & 2 & 1.127 & 0.056 & 16 & $\ldots$ \\
\hline $2 \mathrm{M} 16315358-1302179$ & M 107 & RGB & 5080 & 2.93 & -1.069 & 0.133 & 0.009 & 0.286 & 2 & $<0.666$ & $\ldots$ & 1 & $\ldots$ \\
\hline 2M16315926-1303045 & M 107 & RGB & 5031 & 2.91 & -0.999 & 0.063 & -0.081 & 0.102 & 1 & $<1.726$ & $\cdots$ & 1 & $\ldots$ \\
\hline 2M16320382-1308186 & M 107 & RGB & 5009 & 2.93 & -1.065 & 0.086 & 0.076 & 0.33 & 3 & $<1.445$ & $\ldots$ & 1 & $\ldots$ \\
\hline 2M16320891-1300161 & M 107 & RGB & 4773 & 2.19 & -0.941 & 0.074 & -0.03 & 0.124 & 5 & $<0.648$ & $\cdots$ & 1 & $\cdots$ \\
\hline 2M16320904-1302270 & M 107 & RGB & 5003 & 2.66 & -0.953 & 0.026 & -0.322 & 0.196 & 2 & 1.435 & 0.051 & 14 & $\ldots$ \\
\hline 2M16321394-1301086 & M 107 & RGB & 4307 & 1.55 & -1.044 & 0.021 & -0.172 & 0.027 & 4 & 0.354 & 0.058 & 13 & $\cdots$ \\
\hline 2M16322086-1302131 & M 107 & RGB & 4937 & 2.65 & -0.826 & 0.062 & $<-0.057$ & $\ldots$ & 1 & $<1.728$ & $\ldots$ & 1 & $\ldots$ \\
\hline a & $\ldots$ & $\ldots$ & $\ldots$ & $\ldots$ & $\ldots$ & $\ldots$ & $\ldots$ & $\ldots$ & $\ldots$ & $\ldots$ & $\ldots$ & $\ldots$ & $\ldots$ \\
\hline
\end{tabular}

Notes. The full table is available at the CDS. ${ }^{(*)}$ AGB are distinguished from HB stars such that $T_{\text {eff }}<5300 \mathrm{~K}$.

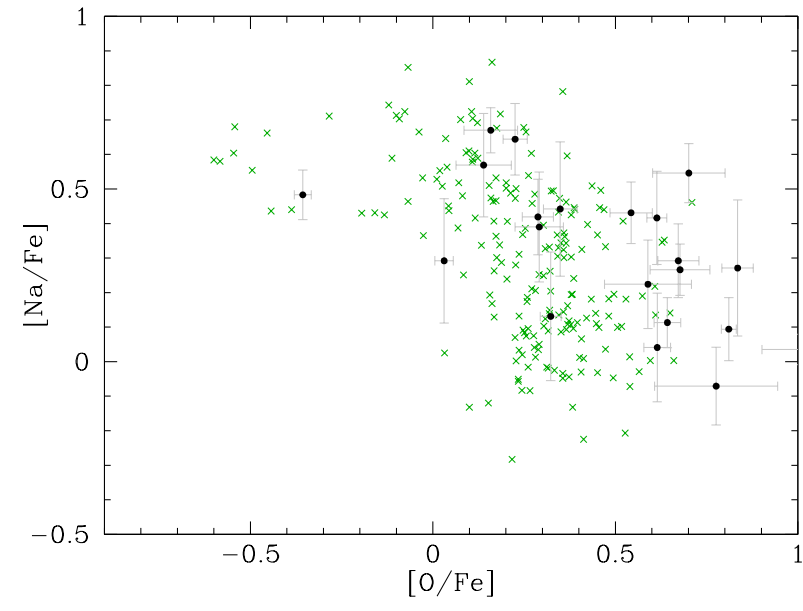

Fig. 11. Na abundances as functions of $\mathrm{O}$ (black points). Although there are only few stars in our sample where $\mathrm{Na}$ could be measured, the $\mathrm{Na}$ and $\mathrm{O}$ abundances follow the expected anticorrelation as measured by Carretta et al. (2009b; green crosses) in the optical.

clusters (hence mostly M71 and M 107 and very few in M 5, $\mathrm{M} 3$, and M 13). Given that $\mathrm{O}$ is also difficult to measure for the warmest stars, our version of the $\mathrm{O}-\mathrm{Na}$ anticorrelation diagram (Fig. 11) appears unpopulated. Nevertheless, the values are consistent with very high resolution optical studies (Carretta et al. $2009 \mathrm{~b}$ ) and thus confirm the high quality of the APOGEE data and of our analysis.

However, the $H$ band contains very clear $\mathrm{Al}$ I lines that are more easily measurable than the $\mathrm{Na} I$ lines. Therefore, Al represents a more robust abundance indicator in the APOGEE data for the study of the multiple stellar population phenomenon in GCs, as first presented by Mészáros et al. (2015). Indeed, in Fig. 12 we demonstrate that we can observe the Al-O anticorrelation for the ten clusters separately, except for M 107 and M 71. Given that the two latter GCs are the most metal rich of the sample, this may indicate that the temperature conditions in the polluters of those two clusters are too low to efficiently produce Al, as illustrated by Ventura et al. (2016) and Dell'Agli et al. (2018) in the case of massive AGB star polluters.

\section{2. $\mathrm{Mg}, \mathrm{Al}$, and $\mathrm{Si}$}

In Fig. 13, we establish the Mg-Al anticorrelation for all clusters as already found in the literature (except M 107 and M 71 as already noted by Mészáros et al. 2015). This implies that the $\mathrm{Mg}-\mathrm{Al}$ chain is active in almost all the clusters (including M 5) in contrast to the conclusions of Carretta et al. (2009a). An interesting aspect shown in these data when scrutinizing those diagrams is that stars with $[\mathrm{Mg} / \mathrm{Fe}]<0$ in $\mathrm{M} 15$ and $\mathrm{M} 92$ seem to have extremely depleted $\mathrm{Mg}$ and lower $\mathrm{Al}$ abundances than expected from the extension of the $\mathrm{Mg}-\mathrm{Al}$ anticorrelation for stars with such low $[\mathrm{Mg} / \mathrm{Fe}]$ abundances. We stress that, regarding any possible analysis bias, we have not been able to find any dependence on effective temperature nor evolutionary status (RGB or eAGB/RHB). Moreover, in Fig. 15 we show the spectra around the two Al I lines present in the APOGEE spectra. This figure unambiguously demonstrates that the $\mathrm{Al} \mathrm{I} \mathrm{lines} \mathrm{are} \mathrm{weaker}$ in the most $\mathrm{Mg}$-depleted stars. Thus, we conclude that the relatively low $\mathrm{Al}$ in such extremely Mg-depleted stars in M 15 and M92 is real.

The Al-Si plane can be seen in Fig. 14. Overall Si is constant and consistent with field star value for similar metallicities. But three clusters (M15, M 92, and M 13) show a significant Si enhancement for the Al richest stars. Yong et al. (2005), Carretta et al. (2009a), and Mészáros et al. (2015) interpreted the Al-Si correlation as a signature of ${ }^{28} \mathrm{Si}$ leakage from the $\mathrm{Mg}$ Al chain. Interestingly, most of the Si enriched stars in M 15 and M92 also seem to correspond to the extreme Mg-depleted and mildly enhanced $\mathrm{Al}$ stars mentioned above. According to Prantzos et al. (2017) during H-burning processes, Si begins to be produced above $80 \mathrm{MK}$. According to the same authors ${ }^{27} \mathrm{Al}$ is expected to be progressively produced up to $\sim 80 \mathrm{MK}$ but begins 


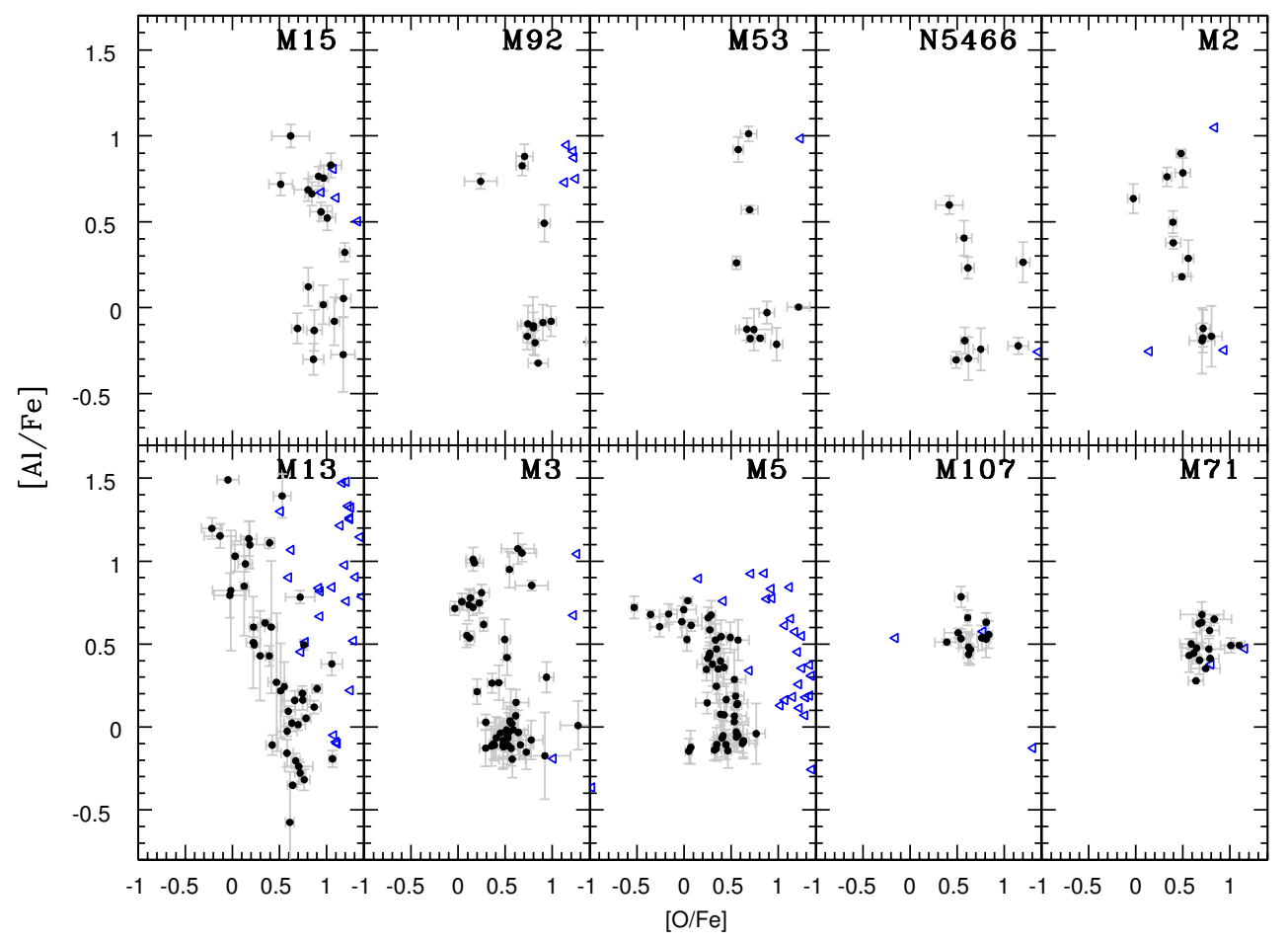

Fig. 12. Al abundances as functions of $\mathrm{O}$ for all GCs in our sample. Black dots indicate measurements and blue triangles indicate upper limits in $\mathrm{O}$. The anticorrelation between those elements can be clearly seen for all clusters individually, except M 107 and M71.

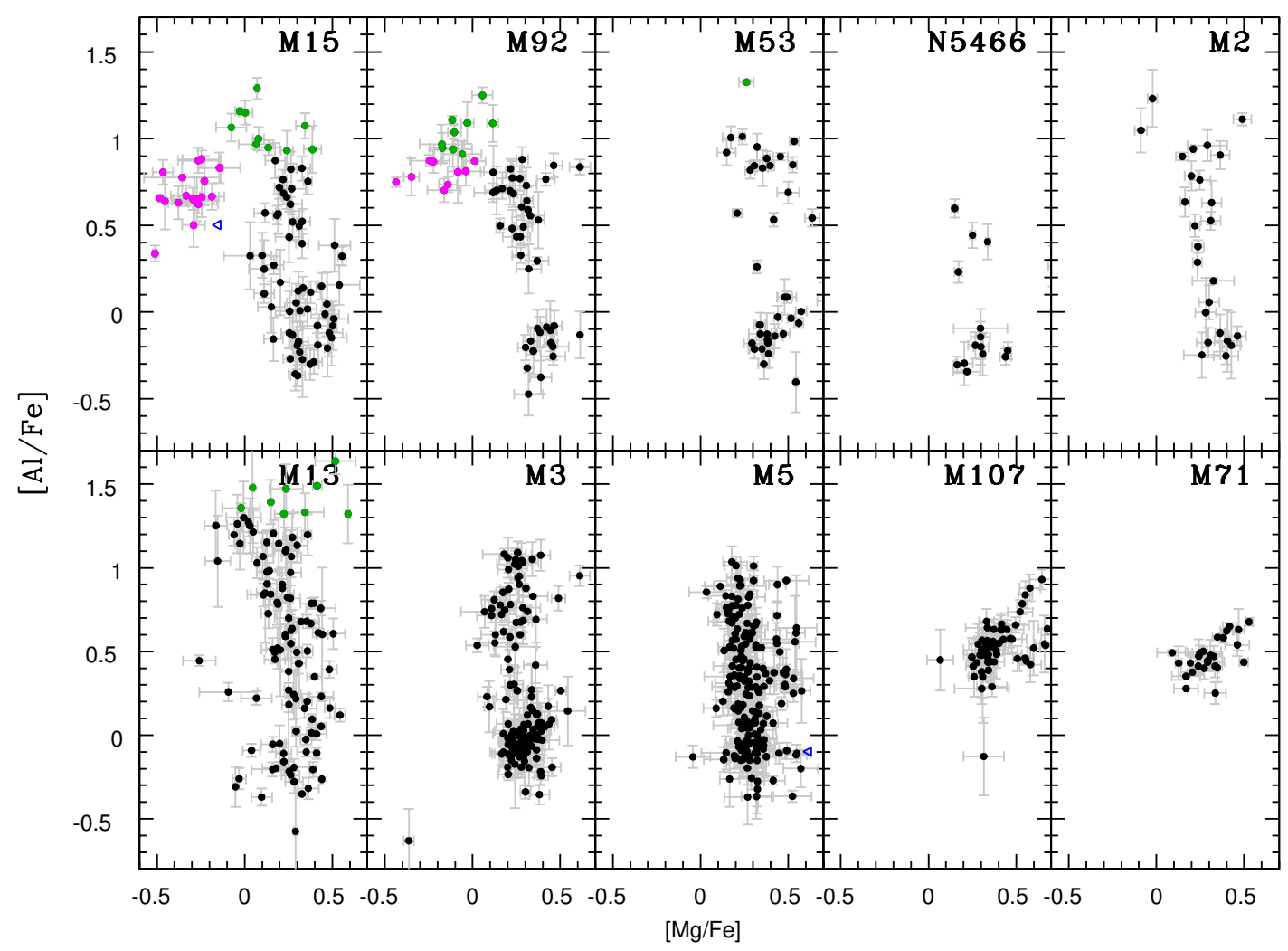

Fig. 13. Al abundances as functions of $\mathrm{Mg}$ abundances for all GCs in our sample. The green points show the Si- and Al-enhanced stars from Fig. 14. Magenta points highlight the extreme Mg-depleted stars. These stars also show a relatively lower $\mathrm{Al}$ abundance.

to be depleted above $80 \mathrm{MK}$. Therefore, temperatures in polluters above $80 \mathrm{MK}$ would explain satisfactorily the existence of Mg- and Al-weak (and Si enhanced) stars in M 15 and M92.

Interestingly, M 13 also shows at least as high $\mathrm{Al}$ enhancements as M 15 and M $92([\mathrm{Al} / \mathrm{Fe}]>1)$. While only a few stars may show some $\mathrm{Si}$ enhancement, none of these appear to be extremely $\mathrm{Mg}$-depleted and weakly enhanced in $\mathrm{Al}$ such as in M 15 and M 92. Knowing that M 13 is more metal rich than M 15 and M92, this may suggest that there is a metallicity dependence on the production/depletion yields of the $\mathrm{Al}$ source.

Finally, if we now consider the whole sequence formed by the Mg-depleted stars with the more standard $\mathrm{Mg}-\mathrm{Al}$ 


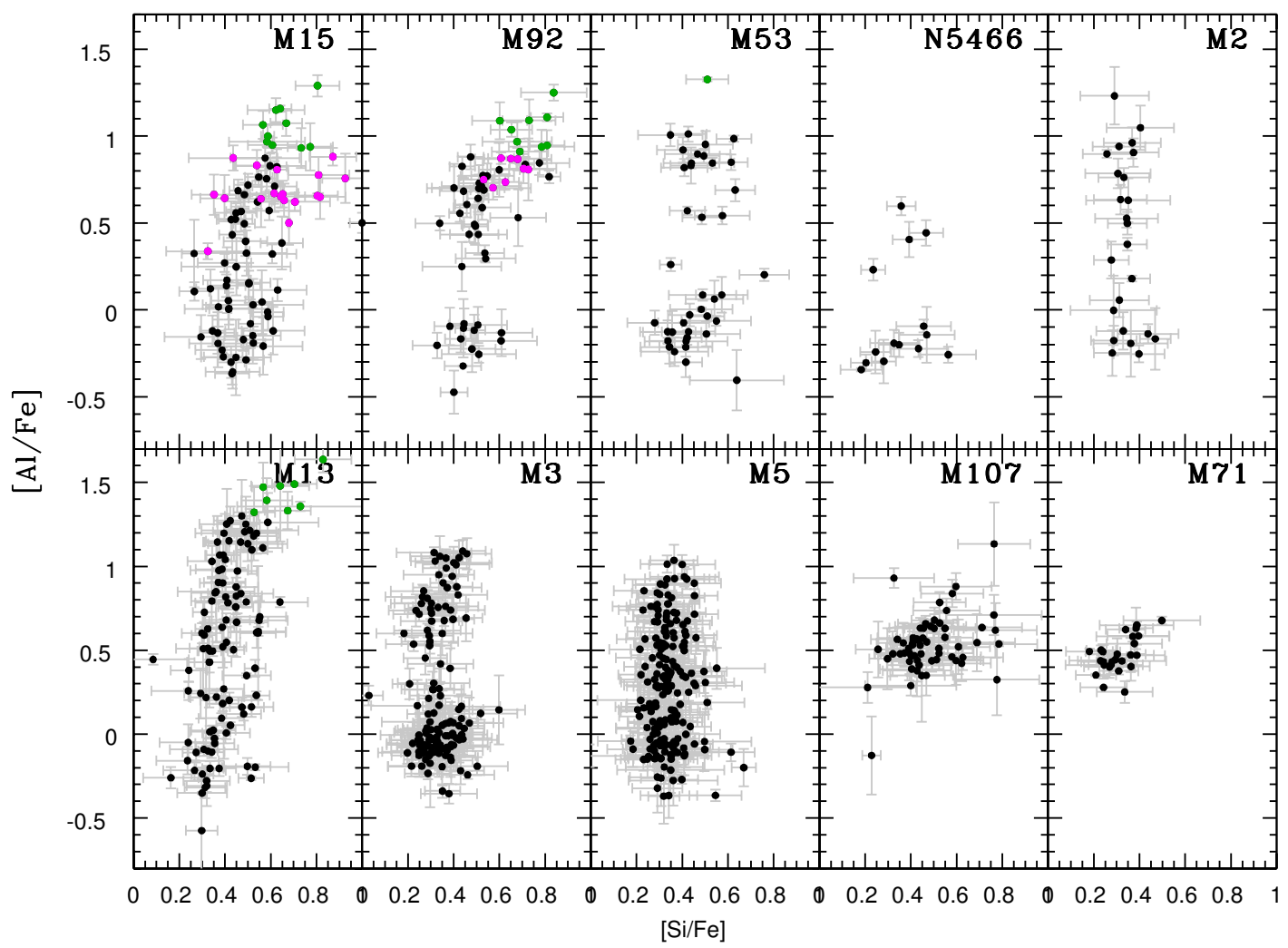

Fig. 14. $\mathrm{Al}$ abundances as functions of $\mathrm{Si}$ abundances for all GCs in our sample. The magenta points highlight the very low $\mathrm{Mg}$ and $\mathrm{Al}$-weak stars from Fig. 13. The green points show the Si- and Al-enhanced stars. There is generally no correlation and $\mathrm{Si}$ is very homogeneous except in M 15 , M92, and possibly M 13 .

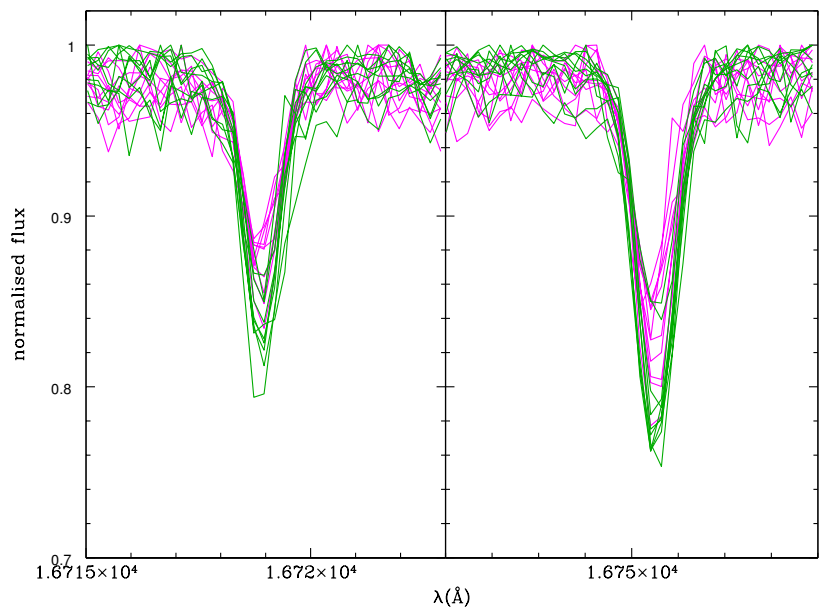

Fig. 15. Al I lines in M15 stars. The green lines show the Si-enhanced stars from Fig. 14, and the magenta lines show the Mg- and Al-weak stars from Fig. 13.

anticorrelation, its appearance is similar to a hook, because the most Mg-depleted stars have smaller than expected Al abundances. Although our data are certainly one of the most extensive spectroscopic studies of those clusters, we may still be missing some data, and the difficulty of analyzing such low-metallicity stars may result in biases in the trends. On the other hand, if the most Mg-poor stars indeed have lower than expected Al abundances, this would imply that standard correlation and anticorrelation patterns observed in GCs are in fact more complex than previously thought. This is well in line with the recent partitioning of the clusters photometric map (the so-called chromosome map) into various subpopulations by Milone et al. (2017) and in particular the discovery of the puzzling extension of the first population by Lardo et al. (2018).

\section{3. $C$ and $N$}

In Fig. 16, the $\mathrm{C}$ and $\mathrm{N}$ anticorrelation can be observed for most of the clusters except the most metal-poor clusters in which $\mathrm{CO}$ and $\mathrm{CN}$ lines are too weak to be detected. We first wondered about the influence of the intrinsic RGB extra-mixing over the $\mathrm{C}$ and $\mathrm{N}$ data. Indeed, the existence of the extra-mixing and its $\mathrm{C}$ and $\mathrm{N}$ signature is clearly seen in the APOGEE field stars (Masseron et al. 2017; Shetrone, in prep.) and can have a very large impact on the $\mathrm{C}$ and $\mathrm{N}$ yields at very low metallicity. We plot in Fig. 17 the $\mathrm{C} / \mathrm{N}$ ratios over the effective temperature and compare these ratios to the model expectations using a prescription for the extra-mixing from Lagarde et al. (2012). This model predicts a significant drop of the $\mathrm{C} / \mathrm{N}$ ratio after the bump luminosity around $4700 \mathrm{~K}$. However, it is difficult to evaluate the impact of the extra-mixing on the $\mathrm{C}$ and $\mathrm{N}$ yields by comparing the $\mathrm{C}$ and $\mathrm{N}$ abundances before and after the luminosity bump because the $[\mathrm{C} / \mathrm{N}]$ data show a very large scatter. Indeed, it is very likely that the extra-mixing signature also depends on the initial abundances of $\mathrm{C}$ and $\mathrm{N}$, which have been proven to vary greatly in GCs from the observation of unevolved stars (see Gratton et al. 2004, and references therein). Consequently, we stress that, in contrast to the work of Lagarde et al. (2019), only field stars represent reliable test beds for studying extra-mixing along the RGB but no GCs stars should be used as test beds.

However, extra-mixing is believed to induce $\mathrm{CN}$-cycling, i.e., that extra-mixing affects $\mathrm{C}$ and $\mathrm{N}$ yields but not the $\mathrm{C}+\mathrm{N}+\mathrm{O}$ yields. By looking at the $\mathrm{C}+\mathrm{N}+\mathrm{O}$ yields we can verify whether 


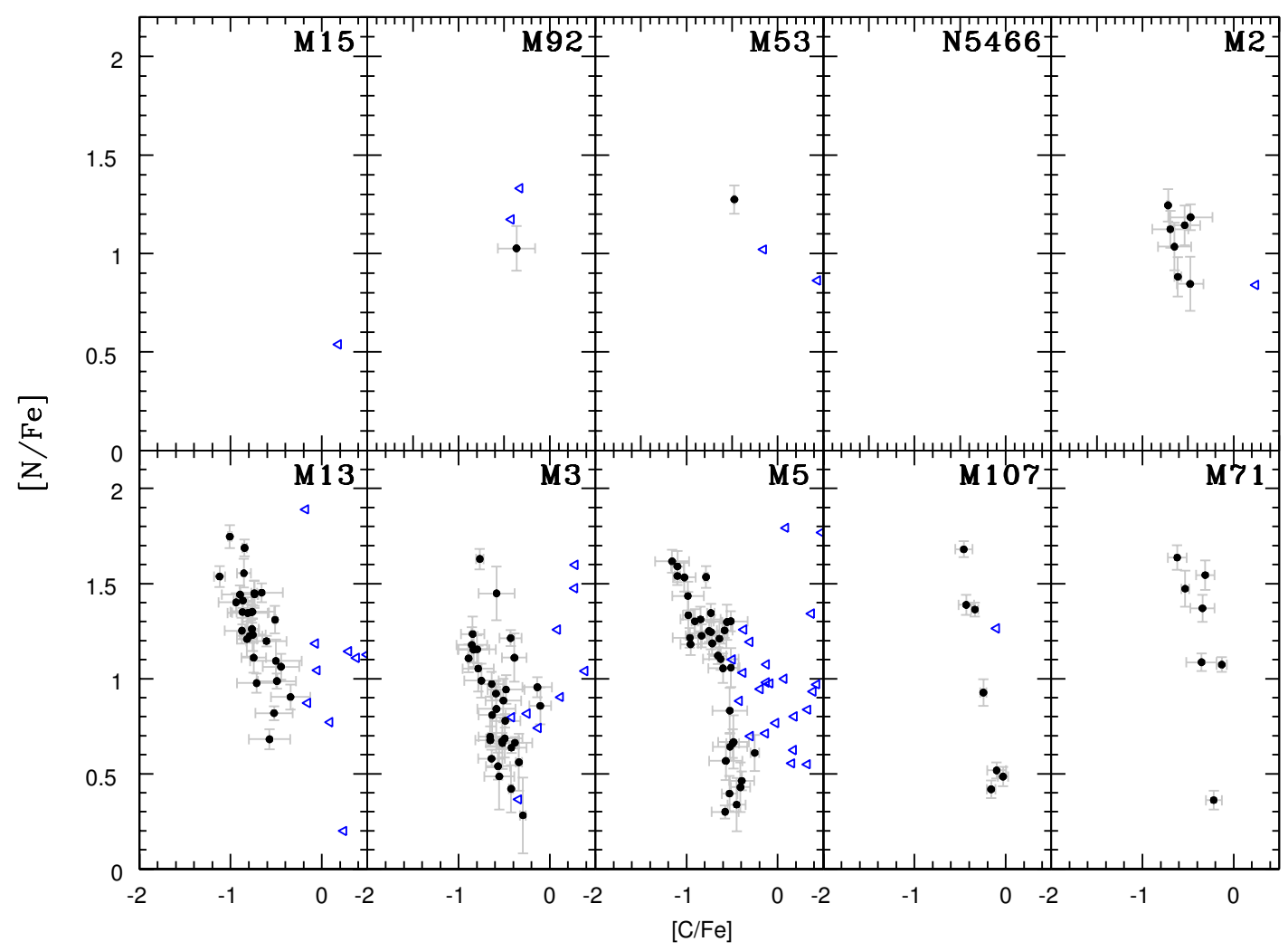

Fig. 16. $\mathrm{N}$ abundances as functions of $\mathrm{C}$ abundances for all GCs in our sample. Black dots indicate measurements and blue triangles indicate upper limits in $\mathrm{C}$. The $\mathrm{N}-\mathrm{C}$ anticorrelation is clearly observed for the most metal-rich clusters (bottom panels).

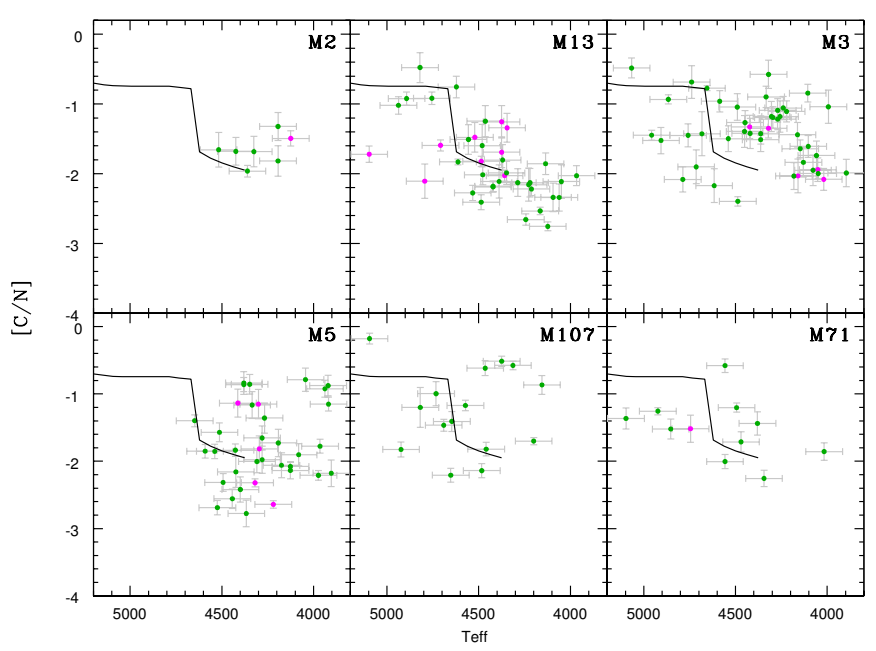

Fig. 17. $[\mathrm{C} / \mathrm{N}]$ ratios as functions of effective temperature for the most metal-rich GCs of our sample. RHB/eAGB stars are shown in magenta, while RGB stars are shown in green. The data are compared with a Lagarde et al. (2012) $0.85 M_{\odot} Z=0.0001$ model (solid black line). This model has been shifted by -0.5 in $\mathrm{C} / \mathrm{N}$ to match approximately the post-first dredge up $\mathrm{C} / \mathrm{N}$ data value.

the $\mathrm{C}+\mathrm{N}+\mathrm{O}$ is varying in a cluster, and thus constrain the nucleosynthesis of the cluster polluters independently of extra-mixing effect. We checked the $\mathrm{C}+\mathrm{N}+\mathrm{O}$ values in our clusters. Compared to Mészáros et al. (2015), it appears very clear now that no correlation exists between $\mathrm{C}+\mathrm{N}+\mathrm{O}$ and $\mathrm{Al}$ for at least three clusters (M 13, M 3 and M 5). Furthermore, the $\mathrm{C}+\mathrm{N}+\mathrm{O}$ data are consistent with no variations within the errors and are also consistent with field stars values of similar metallicities (e.g., Gratton et al.
2000). Therefore, the CNO cycle is also occurring in the GCs polluters. Moreover, we do not find significant enhancement in any of our clusters (including those with upper limits) compared to the value of field stars such as has been claimed by Yong et al. (2009, 2015) in NGC 1851, although disputed by Villanova et al. (2010).

\subsection{Correlations with cluster global properties}

In the previous sections, we confirmed that $\mathrm{Al}$ is (anti)correlated with many elements (C, N, O, Na, Mg, and Si). Given the completeness of the $\mathrm{Al}$ measurements in our sample and its large variations, we consider this element as the best representation to evaluate the extent of the multiple populations of the clusters. As already suggested by Carretta et al. (2009b; but see also Mészáros et al. 2015; Ventura et al. 2016; Dell'Agli et al. 2018), Al spread within each cluster decreases with increasing cluster metallicity. Thanks to our large sample, we can now attempt to compare quantitatively this spread against clusters metallicities and other global properties. In Fig. 18, we plot the Al spread (derived from the deviation from the median) against cluster metallicity, absolute magnitude, and mass. We note that M 15 and M 92 probably have lower than expected $\mathrm{Al}$ abundances in the most extreme cases. Therefore, the Al spread may be underestimated for those clusters. We also remark that NGC 5466 has a quite low number of stars, thus we also probably underevaluate the real Al spread. The diagrams presented Fig. 18 suggest an anticorrelation between the Al spread and the metallicity and a possible correlation between the Al spread and absolute magnitude. From a nucleosynthetic point of view, this potentially implies that the $\mathrm{Mg}-\mathrm{Al}$ chain reaction is becoming less important with increasing metallicity and/or cluster luminosity. Moreover, although cluster absolute luminosity is known to be a proxy 

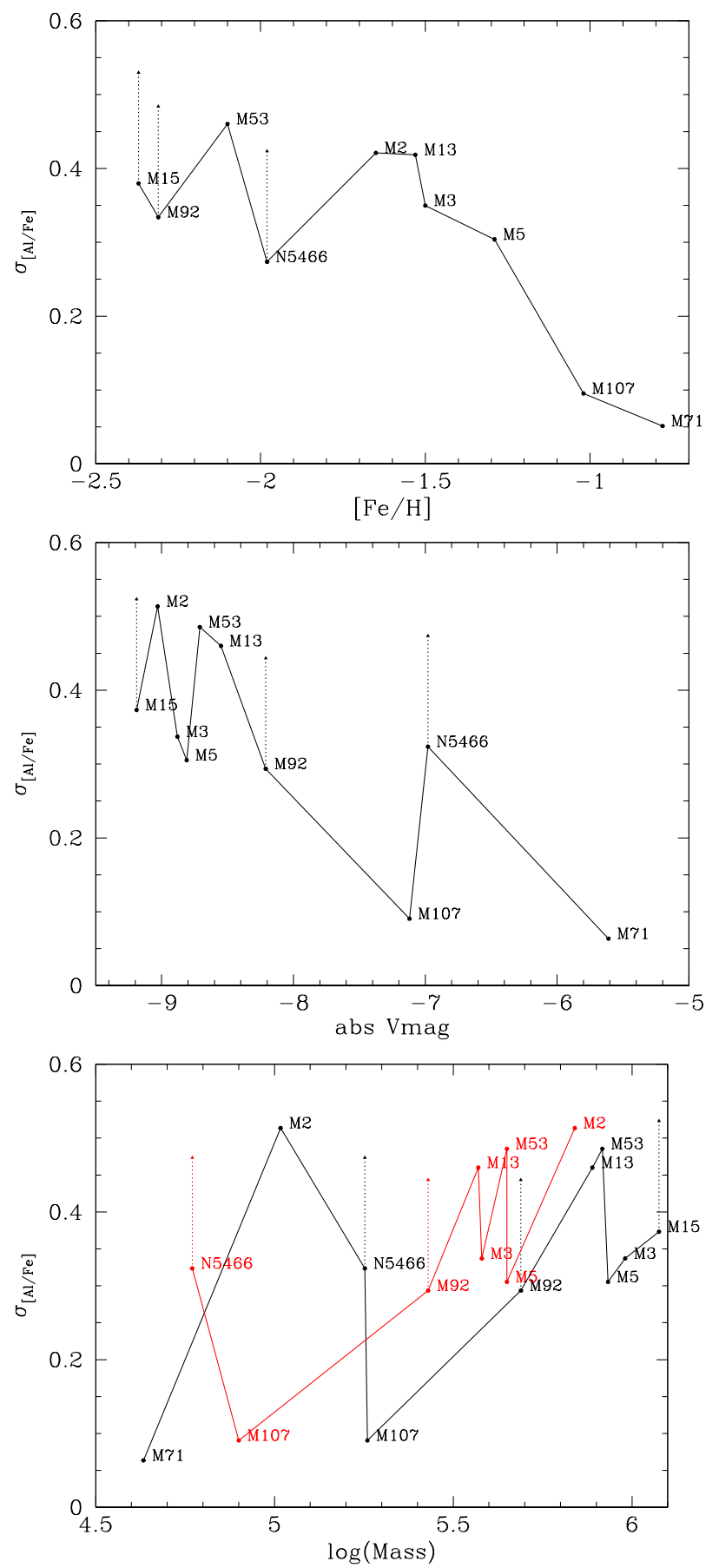

Fig. 18. Spread of $\mathrm{Al}$ as a function of global cluster parameters (metallicity, absolute magnitude, and total masses). M 15, M92, and NGC 5466, which probably have an underestimated spread, are highlighted accordingly. The metallicity and absolute magnitudes are extracted from Harris (2010). The total masses are computed by McLaughlin \& van der Marel (2005; black line) and from a compilation by Boyles et al. (2011; red line). There is probably an anticorrelation with metallicity and maybe a correlation with absolute magnitude.

for cluster mass, we found that the correlation of the Al spread with cluster mass is very uncertain, mostly because cluster mass determination is model dependent.

Similarly, Carretta et al. (2009b) found a bilinear anticorrelation between their $\mathrm{Na}_{\max }(\propto \mathrm{Na}$ spread) and clusters luminosities and metallicities. This is consistent with our results because $\mathrm{Al}$ and $\mathrm{Na}$ have been demonstrated to be correlated (Carretta et al. 2009a). But this contrasts with Milone et al. (2017) who rather found a correlation between metallicity - as well as cluster magnitude - and the width of the RGB. This apparent contradiction could be explained by the fact that the RGB width in a colormagnitude diagram is known to be sensitive to $\mathrm{N}$ because of molecular bands (but not to $\mathrm{Al}$ which has no molecular bands). Thus, we can deduce that in Milone et al. (2017), the N spread is increasing with metallicity. But this phenomenon is difficult to confirm with our data (Fig. 19) because $\mathrm{N}$ is measured in only a fraction of the stars of our sample, statistically weakening the possibility of measuring accurately the $\mathrm{N}$ spread. Actually, in all the clusters in which we were able to measure $\mathrm{N}$ in a large enough number of stars (M 13, M 3, M 5, M 107, and M 71), the $\mathrm{N}$ spread seems rather constant $(\sim 1.5 \mathrm{dex})$ except for the most metal-poor spread M 2 where it is nearly null.

\section{5. $K$}

Although the exact temperature for the onset of the $\mathrm{K}$ production by H-burning nucleosynthesis may be still debated (120-180 MK Ventura et al. 2012; Iliadis et al. 2016), the temperature must be much higher than that for the onset of $\mathrm{Al}$ or Si production $(\sim 80 \mathrm{MK})$. We certainly observe Si production in M 15 and M 92 (Fig. 14), indicating that in these clusters the polluters had reached the highest temperatures. But a variation in $\mathrm{K}$ is observed neither in those extreme clusters nor in the other clusters (Fig. 20). We conclude that the K measured in those clusters is consistent with no $\mathrm{K}$ production, thus relatively lowtemperature nucleosynthesis conditions (but still high enough to produce $\mathrm{Si}$ ), and that $\mathrm{K}$ production in GCs is rare, which agrees in with the conclusions of Takeda et al. (2009) and Carretta et al. (2013). Still, it has been clearly established by Cohen \& Kirby (2012) and Mucciarelli et al. (2015a) that NGC 2808 and NGC 2419 show K enhancement correlated with notably Al. It is remarkable that these two clusters have metallicities in the same range as our sample. Therefore, in contrast to Si production (Sect. 4.2) or Al spread (Sect. 4.4), K production in GCs is not only a function of metallicity. In any case, the absolute luminosities of those K-enhanced clusters are among the largest. This may corroborate the idea that the peculiar chemistry of the clusters is not only a function of metallicity but also a function of cluster luminosity (Carretta et al. 2009a; Milone et al. 2017)

\section{6. $\mathrm{Ca}$}

Marino et al. (2009) observed a Ca spread in M22. But Ca is not expected to be affected by H-burning processes (Prantzos et al. 2017). Fig. 21 shows the $\mathrm{Ca}$ abundances measured in our ten GCs. It is clear in that figure that no correlation with $\mathrm{Al}$ is observed. The star-to-star scatter is also small enough to show no enhancement in any of the clusters. Therefore, M 22 certainly remains an exceptional cluster regarding $\mathrm{Ca}$ enhancement, although Mucciarelli et al. (2015b) suspect that NLTE effects could be also responsible for such an observational spread.

\section{7. s-process elements: $\mathrm{Ce}$ and $\mathrm{Nd}$}

In Fig. 22, we confirm that most GCs do not seem to show a significant spread in Ce nor show any enhancement compared to field stars value as already remarked by Gratton et al. (2004). In contrast, M 15 shows a significant spread. Nd is difficult to measure and we obtain some constraining values only for the most favorable spectra, i.e., in the coolest giants of the most metal-rich GCs. We still observe a consistent correlation with Ce (Fig. 23). Unfortunately, with only one neutroncapture element measured in M 15, it is not possible to assign the 


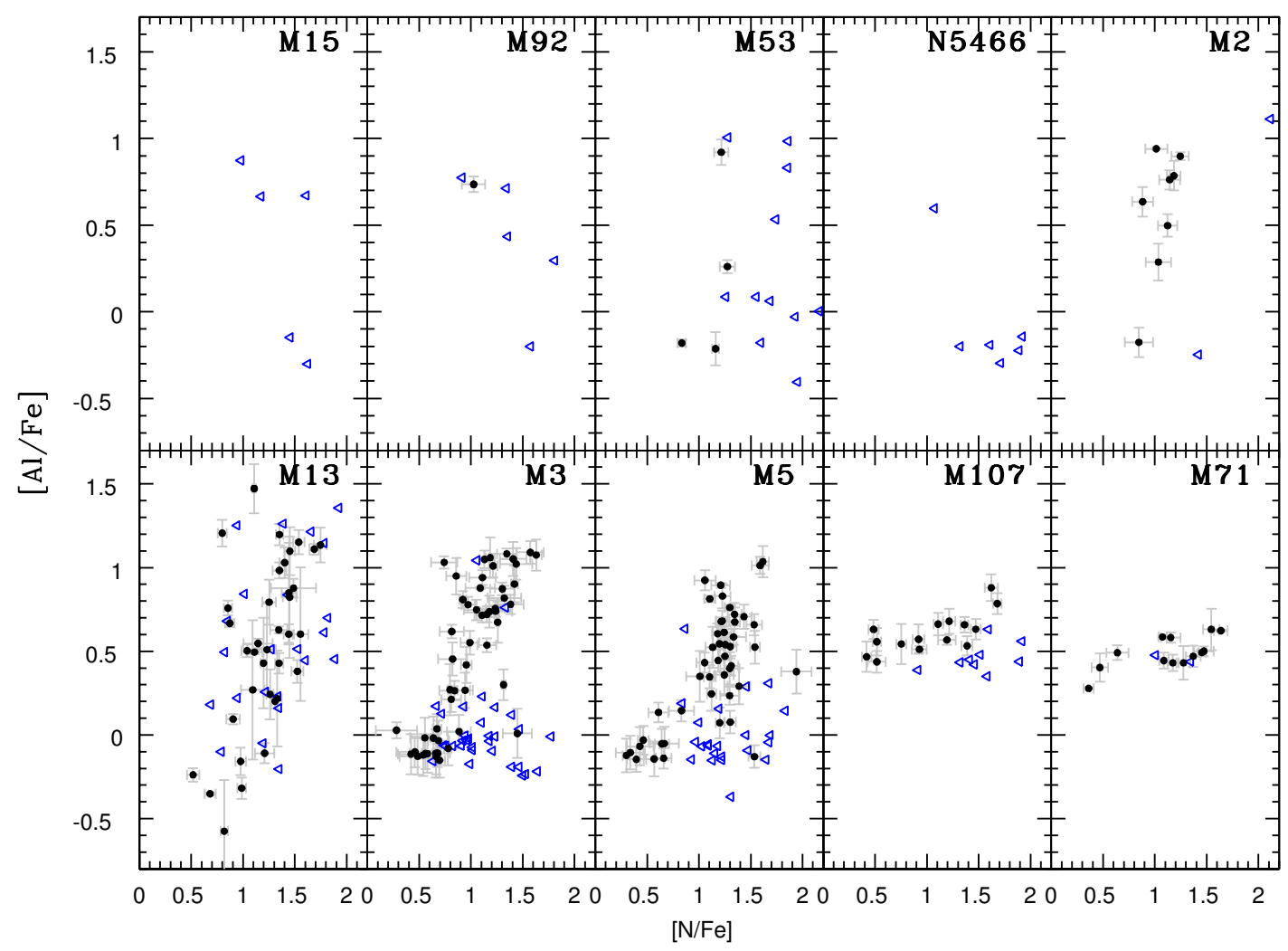

Fig. 19. $\mathrm{Al}$ abundances as functions of $\mathrm{N}$ abundances for all the clusters sorted by metallicity. Blue triangles indicate upper limits in $\mathrm{N}$. Al and $\mathrm{N}$ are correlated, but the $\mathrm{Al}$ spread is decreasing with increasing metallicity.

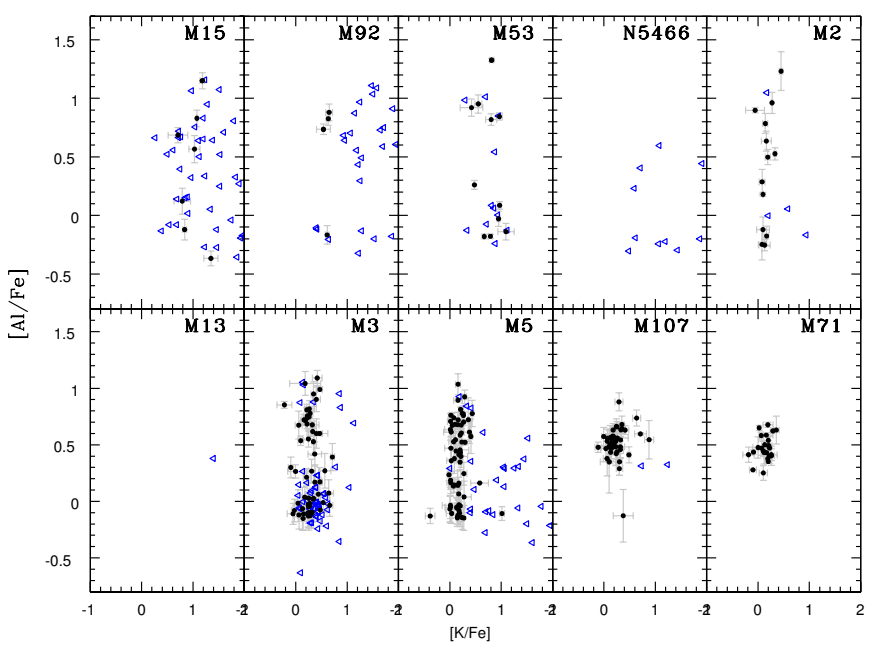

Fig. 20. $\mathrm{Al}$ abundances as functions of $\mathrm{K}$ abundances for all $\mathrm{GCs}$ in our sample. Blue triangles indicate upper limits in $\mathrm{K}$. While $\mathrm{K}$ is difficult to measure in very low-metallicity GCs, there is no significant spread in the remaining GCs. M 13 does not contain measurements because the $\mathrm{K}$ lines at the cluster doppler shift fall in a APOGEE CCD gap where no measurement can be made.

nucleosynthetic origins in those stars, and notably disentangle an $r$-process and $s$-process nucleosynthesis such as discussed in M 22 and M 2 (Roederer \& Sneden 2011; Yong et al. 2014). Therefore, our conclusions concerning M 15 are bound to Sobeck et al. (2011), i.e., that the Ce we measured probably has a pure $r$-process origin. We also agree that the Ce dispersion in M 15 is comparable to that of the halo field at the same metallicity, although we have now clearly established that M 15 also shows large Al variation. But we hardly see a correlation with $\mathrm{Al}$, confirming that polluters in our GCs do not produce $\mathrm{Ce}$ and that $\mathrm{Ce}$ is probably from the GCs primordial formation gas.

\section{Conclusions}

We confirm that APOGEE spectra provide precise elemental abundances for many elements. However, we show that the latest data release (DR14) still suffers from uncertainties, in particular regarding extreme abundances such as those found in some cases for $\mathrm{C}$ and $\mathrm{N}$, which has already been pointed out by Jönsson et al. (2018), but more generally for very low metallicity spectra $([\mathrm{Fe} / \mathrm{H}]<-1.5)$ in which metallic lines become very weak and are extremely sensitive to choice of prescription for parameters like macroturbulence and NLTE effects. We emphasize the crucial lack of an upper limits flagging system fro APOGEE. Finally, we demonstrate that the ASPCAP DR $14 T_{\text {eff }}$ are probably biased whenever $[\mathrm{O} / \alpha]$ are not solar.

With our independent analysis, we measure almost all the elemental abundances needed for GCs studies, except He and $\mathrm{Na}$; the neutron-capture elements $\mathrm{Ce}$ and $\mathrm{Nd}$ are newly included in this survey. Although those latter two elements abundances would certainly be very interesting to be measured as well, we demonstrate that all known light elements anticorrelation can be recovered with a high precision. Consequently, we confirm that $\mathrm{H}$-burning reactions are the main nucleosynthesis processes that have occurred in all the clusters in this analysis.

Moreover, along with literature, we collected some corroborating evidence suggesting that cluster luminosity and metallicity are the two main parameters that drive the various GCs chemical patterns. Unfortunately, our sample of GCs is such that we can hardly disentangle the main factor controlling the 


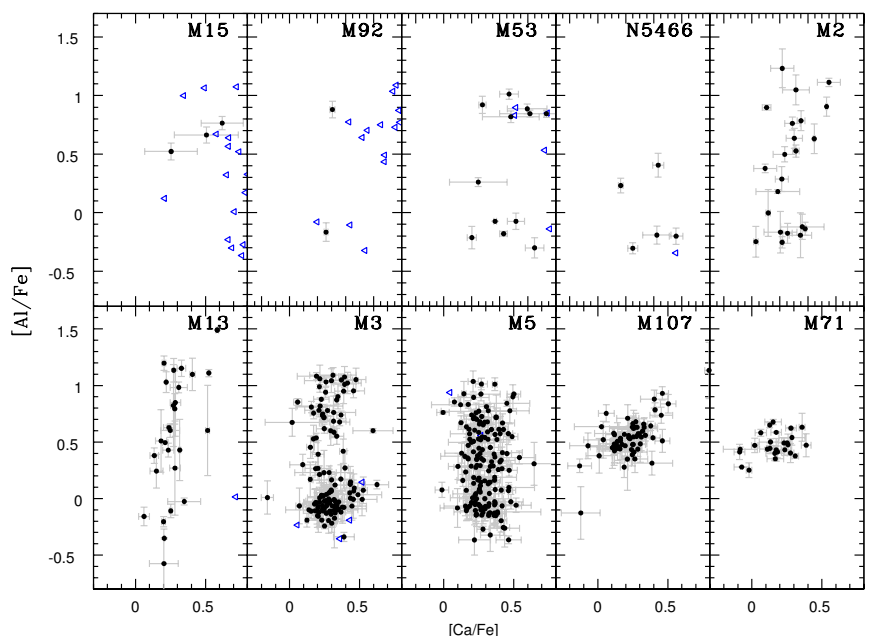

Fig. 21. $\mathrm{Al}$ abundances as functions of $\mathrm{Ca}$ abundances for all $\mathrm{GCs}$ in our sample. Blue triangles indicate upper limits in $\mathrm{Ca}$. While $\mathrm{Ca}$ is difficult to measure in very low metallicity GCs, there is no significant spread in any of the clusters.

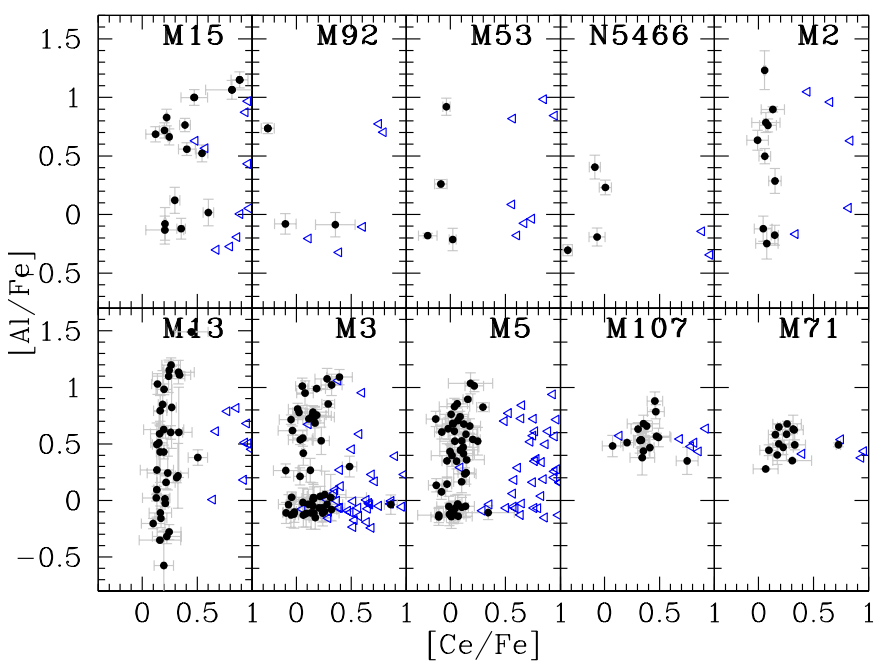

Fig. 22. Al abundances as functions of $\mathrm{Ce}$ abundances for all GCs in our sample. Black dots indicate measurements and blue triangles indicate upper limits in Ce. Except for M 15 (and perhaps M92), all clusters are consistent with a homogeneous $\mathrm{Ce}$ abundance.

amplitude of the pollution between metallicity and cluster magnitude. Indeed, in our sample both are correlated, so that the most metal-rich clusters are also the less luminous, and conversely, the most metal-poor clusters are, on average, the brightest clusters. To validate those main dependencies, it will be interesting to analyze more peculiar clusters such as NGC 2808, NGC 2419, M 2, M 4 M 22, NGC 1851, $\omega$ Cen, or any young massive stellar clusters planned to be observed by the ongoing SDSS IV/APOGEE2 survey.

Furthermore, we discovered some puzzling stars extremely depleted in $\mathrm{Mg}$ and weakly enhanced in $\mathrm{Al}$ that seem to occur only in our most metal-poor clusters, suggesting that the temperature conditions reached in the corresponding polluters are high enough to start burning Al. Finally, in the same $\mathrm{Mg}$-Al plane, we observed that the data are forming a hook. Any model that attempts to explain the multiple populations in GCs must now be able to self-consistently account for such a turnover in the $\mathrm{Mg}-\mathrm{Al}$ anticorrelation as seen in these new detailed and extensive observations.

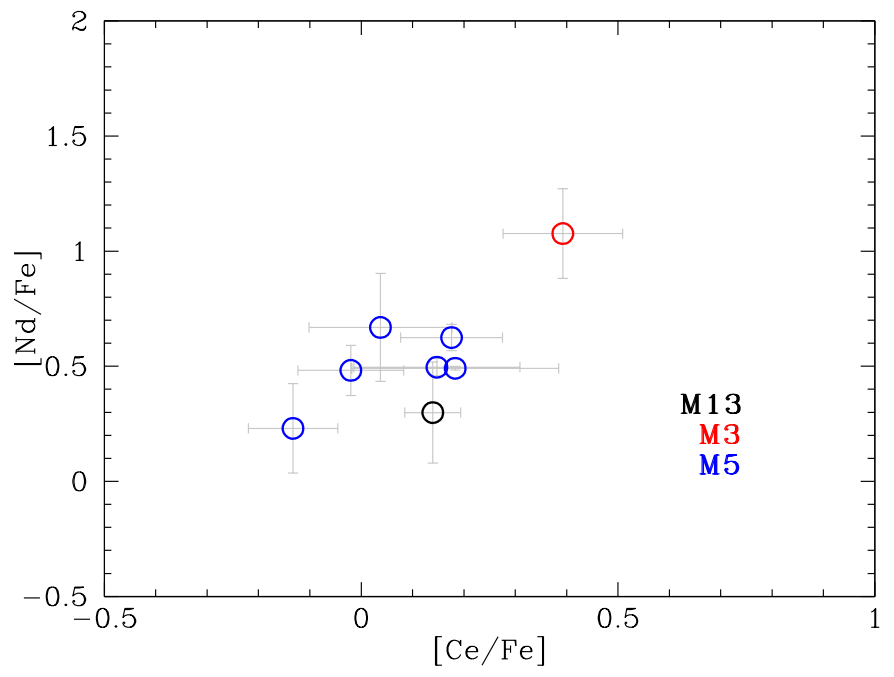

Fig. 23. Nd abundances as functions of Ce abundances for all GCs in our sample. Although there are few measurements of $\mathrm{Nd}$, they correlate well with $\mathrm{Ce}$.

Acknowledgements. We are very grateful for the fruitful comments from P. Ventura and the help from S. Villanova. T.M. acknowledges support from Spanish Ministry of Economy and Competitiveness (MINECO) under the 2015 Severo Ochoa Program SEV-2015-0548. T.M., D.A.G.H., O.Z., and F.D.A. also acknowledge support by the MINECO under grant AYA-2017-88254-P. SzM has been supported by the Premium Postdoctoral Research Program of the Hungarian Academy of Sciences, and by the Hungarian NKFI Grants K-119517 of the Hungarian National Research, Development and Innovation Office. H. J. acknowledges support from the Crafoord Foundation, Stiftelsen Olle Engkvist Byggmästare, and Ruth och Nils-Erik Stenbäcks stiftelse. D.G. acknowledges support from the Chilean Centro de Excelencia en Astrofísica y Tecnologías Afines (CATA) BASAL grant AFB-170002. D.G. also acknowledges financial support from the Dirección de Investigación y Desarrollo de la Universidad de La Serena through the Programa de Incentivo a la Investigación de Académicos (PIA-DIDULS). T.C.B. acknowledges partial support from grant PHY 1430152; Physics Frontier Center/JINA Center for the Evolution of the Elements (JINA-CEE), awarded by the US National Science Foundation. This paper made use of the IAC Supercomputing facility HTCondor (http://research. cs.wisc.edu/htcondor/), partly financed by the Ministry of Economy and Competitiveness with FEDER funds, code IACA13-3E-2493. Funding for the Sloan Digital Sky Survey IV has been provided by the Alfred P. Sloan Foundation, the U.S. Department of Energy Office of Science, and the Participating Institutions. SDSS acknowledges support and resources from the Center for High-Performance Computing at the University of Utah. The SDSS website is www.sdss.org. SDSS is managed by the Astrophysical Research Consortium for the Participating Institutions of the SDSS Collaboration including the Brazilian Participation Group, the Carnegie Institution for Science, Carnegie Mellon University, the Chilean Participation Group, the French Participation Group, Harvard-Smithsonian Center for Astrophysics, Instituto de Astrofísica de Canarias, The Johns Hopkins University, Kavli Institute for the Physics and Mathematics of the Universe (IPMU)/University of Tokyo, the Korean Participation Group, Lawrence Berkeley National Laboratory, Leibniz Institut für Astrophysik Potsdam (AIP), Max-Planck-Institut für Astronomie (MPIA Heidelberg), Max-Planck-Institut für Astrophysik (MPA Garching), MaxPlanck-Institut für Extraterrestrische Physik (MPE), National Astronomical Observatories of China, New Mexico State University, New York University, University of Notre Dame, Observatório Nacional/MCTI, The Ohio State University, Pennsylvania State University, Shanghai Astronomical Observatory, United Kingdom Participation Group, Universidad Nacional Autónoma de México, University of Arizona, University of colourado Boulder, University of Oxford, University of Portsmouth, University of Utah, University of Virginia, University of Washington, University of Wisconsin, Vanderbilt University, and Yale University.

\section{References}

Abolfathi, B., Aguado, D. S., Aguilar, G., et al. 2018, ApJS, 235, 42 Ahn, C. P., Alexandroff, R., Allende Prieto, C., et al. 2014, ApJS, 211, 17 Bastian, N., \& Lardo, C. 2018, ARA\&A, 56, 83

Bertelli, G., Girardi, L., Marigo, P., \& Nasi, E. 2008, A\&A, 484, 815 
Blanton, M. R., Bershady, M. A., Abolfathi, B., et al. 2017, AJ, 154, 28 Boyles, J., Lorimer, D. R., Turk, P. J., et al. 2011, ApJ, 742, 51

Brooke, J. S. A., Bernath, P. F., Western, C. M., et al. 2016, J. Quant. Spectr. Radiat., 168, 142

Carretta, E., Bragaglia, A., Gratton, R., \& Lucatello, S. 2009a, A\&A, 505, 139

Carretta, E., Bragaglia, A., Gratton, R. G., et al. 2009b, A\&A, 505, 117

Carretta, E., Bragaglia, A., Gratton, R., D’Orazi, V., \& Lucatello, S. 2009c, A\&A, 508, 695

Carretta, E., Gratton, R. G., Bragaglia, A., et al. 2013, ApJ, 769, 40

Charbonnel, C. 2016, in EAS Pub. Ser., eds. E. Moraux, Y. Lebreton, \& C. Charbonnel, 80, 177

Cohen, J. G., \& Kirby, E. N. 2012, ApJ, 760, 86

Cunha, K., Smith, V. V., Hasselquist, S., et al. 2017, ApJ, 844, 145

D’Antona, F., \& Ventura, P. 2007, MNRAS, 379, 1431

Dell'Agli, F., García-Hernández, D. A., Ventura, P., et al. 2018, MNRAS, 475, 3098

Di Criscienzo, M., Ventura, P., D’Antona, F., Dell'Agli, F., \& Tailo, M. 2018, MNRAS, 479, 5325

Dobrovolskas, V., Kučinskas, A., Bonifacio, P., et al. 2015, A\&A, 576, A128

Fernández-Trincado, J. G., Robin, A. C., Moreno, E., et al. 2016, ApJ, 833, 132

Fernández-Trincado, J. G., Zamora, O., García-Hernández, D. A., et al. 2017, ApJ, 846, L2

Fernández-Trincado, J. G., Zamora, O., Souto, D., et al. 2018, A\&A, submitted [arXiv: 1801.07136$]$

García-Hernández, D. A., Mészáros, S., Monelli, M., et al. 2015, ApJ, 815, L4

García Pérez, A. E., Allende Prieto, C., Holtzman, J. A., et al. 2016, AJ, 151 144

Gilmore, G., Randich, S., Asplund, M., et al. 2012, The Messenger, 147, 25

González Hernández, J. I., \& Bonifacio, P. 2009, A\&A, 497, 497

Gordon, I. E., Rothman, L. S., Hill, C., et al. 2017, J. Quant. Spectr. Radiat., 203, 3

Gratton, R. G., Sneden, C., Carretta, E., \& Bragaglia, A. 2000, A\&A, 354, 169

Gratton, R., Sneden, C., \& Carretta, E. 2004, ARA\&A, 42, 385

Gratton, R. G., Carretta, E., \& Bragaglia, A. 2012, A\&ARv, 20, 50

Gray, D. F. 2008, The Observation and Analysis of Stellar Photospheres (Cambridge University Press)

Gunn, J. E., Siegmund, W. A., Mannery, E. J., et al. 2006, AJ, 131, 2332

Gustafsson, B., Edvardsson, B., Eriksson, K., et al. 2008, A\&A, 486, 951

Harris, W. E. 2010, ArXiv e-prints [arXiv:1012 . 3224]

Hasselquist, S., Shetrone, M., Cunha, K., et al. 2016, ApJ, 833, 81

Hekker, S., \& Meléndez, J. 2007, A\&A, 475, 1003

Holtzman, J. A., Shetrone, M., Johnson, J. A., et al. 2015, AJ, 150, 148

Holtzman, J. A., Hasselquist, S., Shetrone, M., et al. 2018, AJ, 156, 125

Iliadis, C., Karakas, A. I., Prantzos, N., Lattanzio, J. C., \& Doherty, C. L. 2016, ApJ, 818, 98

Jönsson, H., Allende Prieto, C., Holtzman, J. A., et al. 2018, AJ, 156, 126

Kraft, R. P. 1994, PASP, 106, 553

Lagarde, N., Decressin, T., Charbonnel, C., et al. 2012, A\&A, 543, A108

Lagarde, N., Reylé, C., Robin, A. C., et al. 2019, A\&A, 621, A24
Lardo, C., Salaris, M., Bastian, N., et al. 2018, A\&A, 616, A168 Li, G., Gordon, I. E., Rothman, L. S., et al. 2015, ApJS, 216, 15 Majewski, S. R., Schiavon, R. P., Frinchaboy, P. M., et al. 2017, AJ, 154, 94 Marigo, P., Girardi, L., Bressan, A., et al. 2017, ApJ, 835, 77

Marino, A. F., Milone, A. P., Piotto, G., et al. 2009, A\&A, 505, 1099

Marino, A. F., Yong, D., Milone, A. P., et al. 2018, ApJ, 859, 81

Masseron, T., \& Gilmore, G. 2015, MNRAS, 453, 1855

Masseron, T., Merle, T., \& Hawkins, K. 2016, Astrophysics Source Code Library [record ascl:1605.004]

Masseron, T., Lagarde, N., Miglio, A., Elsworth, Y., \& Gilmore, G. 2017, MNRAS, 464, 3021

McLaughlin, D. E., \& van der Marel, R. P. 2005, ApJS, 161, 304

Mészáros, S., Martell, S. L., Shetrone, M., et al. 2015, AJ, 149, 153

Mészáros, S., García-Hernández, D. A., Cassisi, S., et al. 2018, MNRAS, 475, 1633

Milone, A. P., Marino, A. F., Piotto, G., et al. 2015, ApJ, 808, 51

Milone, A. P., Piotto, G., Renzini, A., et al. 2017, MNRAS, 464, 3636

Milone, A. P., Marino, A. F., Renzini, A., et al. 2018, MNRAS, 481, 5098

Monelli, M., Milone, A. P., Stetson, P. B., et al. 2013, MNRAS, 431, 2126

Mucciarelli, A., Bellazzini, M., Merle, T., et al. 2015a, ApJ, 801, 68

Mucciarelli, A., Lapenna, E., Massari, D., et al. 2015b, ApJ, 809, 128

Ness, M., Hogg, D. W., Rix, H.-W., Ho, A. Y. Q., \& Zasowski, G. 2015, ApJ, 808,16

Nidever, D. L., Holtzman, J. A., Allende Prieto, C., et al. 2015, AJ, 150, 173

Norris, J. E., \& Da Costa, G. S. 1995, ApJ, 447, 680

Pancino, E., Romano, D., Tang, B., et al. 2017, A\&A, 601, A112

Plez, B. 2012, Astrophysics Source Code Library [record ascl:1205 .004]

Prantzos, N., Charbonnel, C., \& Iliadis, C. 2017, A\&A, 608, A28

Renzini, A., D’Antona, F., Cassisi, S., et al. 2015, MNRAS, 454, 4197

Roederer, I. U., \& Sneden, C. 2011, AJ, 142, 22

Schiavon, R. P., Zamora, O., Carrera, R., et al. 2017, MNRAS, 465, 501

Shetrone, M., Bizyaev, D., Lawler, J. E., et al. 2015, ApJS, 221, 24

Shingles, L. J., Karakas, A. I., Hirschi, R., et al. 2014, ApJ, 795, 34

Smiljanic, R., Korn, A. J., Bergemann, M., et al. 2014, A\&A, 570, A122

Smith, G. H. 1987, PASP, 99, 67

Sneden, C., Lucatello, S., Ram, R. S., Brooke, J. S. A., \& Bernath, P. 2014, ApJS, 214, 26

Sobeck, J. S., Kraft, R. P., Sneden, C., et al. 2011, AJ, 141, 175

Szécsi, D., \& Wünsch, R. 2019, ApJ, 871, 20

Takeda, Y., Kaneko, H., Matsumoto, N., et al. 2009, PASJ, 61, 563

Ventura, P., D'Antona, F., Di Criscienzo, M., et al. 2012, ApJ, 761, L30

Ventura, P., García-Hernández, D. A., Dell'Agli, F., et al. 2016, ApJ, 831, L17

Villanova, S., Geisler, D., \& Piotto, G. 2010, ApJ, 722, L18

Yong, D., Grundahl, F., Nissen, P. E., Jensen, H. R., \& Lambert, D. L. 2005, A\&A, 438, 875

Yong, D., Grundahl, F., D’Antona, F., et al. 2009, ApJ, 695, L62

Yong, D., Roederer, I. U., Grundahl, F., et al. 2014, MNRAS, 441, 3396

Yong, D., Grundahl, F., \& Norris, J. E. 2015, MNRAS, 446, 3319

Zasowski, G., Cohen, R. E., Chojnowski, S. D., et al. 2017, AJ, 154, 198 